\title{
Understanding star formation in molecular clouds
}

\section{Probability distribution functions of molecular lines in Cygnus X}

\author{
N. Schneider ${ }^{1,2}$, S. Bontemps ${ }^{1}$, F. Motte ${ }^{3}$, V. Ossenkopf ${ }^{2}$, R. S. Klessen ${ }^{4}$, R. Simon ${ }^{2}$, S. Fechtenbaum ${ }^{1}$, F. Herpin ${ }^{1}$, \\ P. Tremblin ${ }^{5}$, T. Csengeri ${ }^{6}$, P. C. Myers ${ }^{7}$, T. Hill ${ }^{8}$, M. Cunningham ${ }^{9}$, and C. Federrath ${ }^{10}$ \\ 1 Université Bordeaux, LAB, CNRS, UMR 5804, 33270 Floirac, France \\ 2 I. Physikalisches Institut, Universität zu Köln, Zülpicher Straße 77, 50937 Köln, Germany \\ e-mail: nschneid@ph1.uni-koeln.de \\ 3 IRFU/SAp CEA/DSM, Laboratoire AIM CNRS - Université Paris Diderot, 91191 Gif-sur-Yvette, France \\ ${ }^{4}$ Universität Heidelberg, Zentrum für Astronomie, Albert-Ueberle Str. 2, 69120 Heidelberg, Germany \\ 5 Maison de la simulation, CEA Saclay, 91191 Gif-sur-Yvette, France \\ ${ }^{6}$ Max-Planck Institut für Radioastronomie, Auf dem Hügel, 53121 Bonn, Germany \\ 7 Harvard-Smithsonian Center for Astrophysics, 60 Garden Street, Cambridge MA 02138, USA \\ 8 Joint ALMA observatory, 15782 Santiago, Chile \\ 9 School of Physics, University of New South Wales, Sydney, NSW 2052, Australia \\ 10 Research School of Astronomy and Astrophysics, The Australian National University, Canberra, ACT 2611, Australia
}

Received 8 August 2015 / Accepted 22 November 2015

\begin{abstract}
The probability distribution function of column density (N-PDF) serves as a powerful tool to characterise the various physical processes that influence the structure of molecular clouds. Studies that use extinction maps or $\mathrm{H}_{2}$ column-density maps $(N)$ that are derived from dust show that star-forming clouds can best be characterised by lognormal PDFs for the lower $N$ range and a power-law tail for higher $N$, which is commonly attributed to turbulence and self-gravity and/or pressure, respectively. While PDFs from dust cover a large dynamic range (typically $N \sim 10^{20-24} \mathrm{~cm}^{-2}$ or $A_{v} \sim 0.1-1000$ ), PDFs obtained from molecular lines - converted into $\mathrm{H}_{2}$ column density - potentially trace more selectively different regimes of (column) densities and temperatures. They also enable us to distinguish different clouds along the line of sight through using the velocity information. We report here on PDFs that were obtained from observations of ${ }^{12} \mathrm{CO},{ }^{13} \mathrm{CO}, \mathrm{C}^{18} \mathrm{O}, \mathrm{CS}$, and $\mathrm{N}_{2} \mathrm{H}^{+}$in the Cygnus $\mathrm{X}$ North region, and make a comparison to a PDF that was derived from dust observations with the Herschel satellite. The PDF of ${ }^{12} \mathrm{CO}$ is lognormal for $A_{v} \sim 1-30$, but is cut for higher $A_{v}$ because of optical depth effects. The PDFs of $\mathrm{C}^{18} \mathrm{O}$ and ${ }^{13} \mathrm{CO}$ are mostly lognormal up to $A_{v} \sim 1-15$, followed by excess up to $A_{v} \sim 40$. Above that value, all CO PDFs drop, which is most likely due to depletion. The high density tracers $\mathrm{CS}_{\text {and }} \mathrm{N}_{2} \mathrm{H}^{+}$exhibit only a power law distribution between $A_{v} \sim 15$ and 400, respectively. The PDF from dust is lognormal for $A_{v} \sim 3-15$ and has a power-law tail up to $A_{v} \sim 500$. Absolute values for the molecular line column densities are, however, rather uncertain because of abundance and excitation temperature variations. If we take the dust PDF at face value, we "calibrate" the molecular line PDF of CS to that of the dust and determine an abundance $[\mathrm{CS}] /\left[\mathrm{H}_{2}\right]$ of $10^{-9}$. The slopes of the power-law tails of the $\mathrm{CS}, \mathrm{N}_{2} \mathrm{H}^{+}$, and dust PDFs are $-1.6,-1.4$, and -2.3 , respectively, and are thus consistent with free-fall collapse of filaments and clumps. A quasi static configuration of filaments and clumps can also possibly account for the observed N-PDFs, providing they have a sufficiently condensed density structure and external ram pressure by gas accretion is provided. The somehow flatter slopes of $\mathrm{N}_{2} \mathrm{H}^{+}$and CS can reflect an abundance change and/or subthermal excitation at low column densities.
\end{abstract}

Key words. ISM: abundances - ISM: clouds - dust, extinction - ISM: molecules - ISM: structure

\section{Introduction}

Probability distribution functions (PDFs) form the basis of many modern theories of star formation (e.g. Krumholz \& McKee 2005; Hennebelle \& Chabrier 2008; Federrath et al. 2008, 2010; Padoan \& Nordlund 2011; Federrath \& Klessen 2012, 2013; Hopkins 2013), and are frequently used to characterise properties of the interstellar medium in simulations (Klessen 2000; Vazquez-Semadeni \& Garcia 2001; Burkhart et al. 2013, Ward et al. 2014). In summary, a PDF is defined as the probability of finding gas within a column-density ${ }^{1}$ range $[N, N+\mathrm{d} N]$. We define $\eta \equiv \ln (N /\langle N\rangle)$, and the quantity $p_{\eta}(\eta)$ then corresponds

\footnotetext{
1 The column density $N$ can be expressed as visual extinction $A_{v}$ with $N\left(\mathrm{H}_{2}\right)=A_{v} 0.94 \times 10^{21} \mathrm{~cm}^{-2} \mathrm{mag}^{-1}$ (Bohlin et al. 1978).
}

to the PDF of $\eta$. For more details on the basic definitions, see Schneider et al. (2015a).

Observationally, PDFs were first obtained from near-IR extinction maps (Lombardi et al. 2008; Kainulainen et al. 2009; Froebrich \& Rowles 2010). With the advent of Herschel ${ }^{2}$, it is now possible to determine dust column-density maps that cover a very large dynamic range from $A_{v}<1$ up to a few hundred $A_{v}$. Studies using this type of map reveal large variations in dust PDFs. Earlier findings (Kainulainen et al. 2009) of a simple lognormal plus power-law tail distribution for star-forming

2 Herschel is an ESA space observatory with science instruments provided by European-led Principal Investigator consortia and with important participation from NASA. 
regions are confirmed (e.g. Schneider et al. 2013, 2015a; Stutz \& Kainulainen 2015), but more complex shapes are also detected (Hill et al. 2011; Schneider et al. 2012, 2015c; Russeil et al. 2013; Alves de Oliveira 2014; Tremblin et al. 2014). The outcome of these works is that (i) all clouds have a distribution for low $A_{v}$ that is best described by a lognormal; (ii) a second peak at low $A_{v}$ can emerge in the case of external pressure, which represents the compressed shell; and (iii) a single or double powerlaw tail is found for star-forming regions, and it is proposed that this is related to gravitational collapse and/or external pressure (either as a phase transition between clumps and interclump gas or stellar feedback).

Only a few studies attempt to make PDFs from molecular line observations. $N\left(\mathrm{H}_{2}\right)$-PDFs of ${ }^{12} \mathrm{CO}$ or ${ }^{13} \mathrm{CO}$ (Goldsmith et al. 2008; Wong et al. 2008; Goodman et al. 2009; Lo et al. 2009; Carlhoff et al. 2013; Schneider et al. 2015b) are often clipped at a certain $A_{v}$ threshold because the lines can become optically thick and CO is depleted. Taking W43 as an example, this threshold for ${ }^{13} \mathrm{CO} 2 \rightarrow 1$ lies between $A_{v}=30$ (for an assumed $T_{\mathrm{ex}}=10 \mathrm{~K}$ ) and $\sim 100$ (for $T_{\mathrm{ex}}=12 \mathrm{~K}$ and correction for high opacity). Assembling a PDF from one or several molecular line tracers with high critical densities such as $\mathrm{CS}, \mathrm{HCO}^{+}$, $\mathrm{HCN}, \mathrm{N}_{2} \mathrm{H}^{+}$etc. has not yet been tried owing to the lack of extended maps. In this study, we use our large dataset of Cygnus $\mathrm{X}$ in the molecular lines of ${ }^{12} \mathrm{CO},{ }^{13} \mathrm{CO}$, and $\mathrm{C}^{18} \mathrm{O} 1 \rightarrow 0, \mathrm{CS}$ $2 \rightarrow 1$, and $\mathrm{N}_{2} \mathrm{H}^{+} 1 \rightarrow 0$ that were obtained with the Five Colleges Radio Astronomy Observatory (FCRAO), and dust columndensity maps of the Cygnus X North region (Hennemann et al. 2012; Schneider et al., in prep.) to produce PDFs from $\mathrm{H}_{2}$ column density that were obtained from molecules and dust. We discuss their properties but also point out the large uncertainties and observational difficulties related to molecular line PDFs.

\section{Observations}

\section{Molecular line data}

We use data from a molecular line survey of ${ }^{12} \mathrm{CO}(115.3 \mathrm{GHz})$, ${ }^{13} \mathrm{CO}(110.2 \mathrm{GHz}), \mathrm{C}^{18} \mathrm{O} 1 \rightarrow 0(109.8 \mathrm{GHz}), \mathrm{CS} 2 \rightarrow 1(98.0$ $\mathrm{GHz})$, and $\mathrm{N}_{2} \mathrm{H}^{+} 1 \rightarrow 0(93.2 \mathrm{GHz})$, which were collected with the FCRAO 14m radiotelescope between 2003 and 2006. The data were obtained using the 32 pixel Second Quabbin Optical Imaging Array (SEQUOIA) in an on-the-fly (OTF) observing mode. The beamwidth of the FCRAO at $93 \mathrm{GHz}$ is $\sim 48^{\prime \prime}$ and at $110 \mathrm{GHz} \sim 45^{\prime \prime}$, while the main beam efficiency at that time was 0.48 . For the present paper, we use maps on a $20^{\prime \prime}$ grid. The data have a mean $1 \sigma_{\text {rms }}$ rms noise level of $\sim 0.4 \mathrm{~K}$ per $0.06 \mathrm{~km} \mathrm{~s}^{-1}$ channel on a $T_{\mathrm{mb}}$ temperature scale that we use in this paper. In the $\mathrm{C}^{18} \mathrm{O}$ map, the noise level and a slightly stripy appearance, resulting from the on-the-fly mapping mode, becomes apparent. However, this does not affect the PDFs because they are assembled from pixels above the $3 \sigma_{\mathrm{rms}}$ level. For further details, see Schneider et al. (2010, 2011).

\section{Column-density maps from Herschel}

For this study, we employ a column-density map at 36" angular resolution (regridded to $20^{\prime \prime}$ to match the fully-sampled FCRAO maps) that were obtained as part of the HOBYS (Herschel imaging survey of OB Young Stellar objects) key program (Motte et al. 2010). This map was obtained by an SED (Spectral Energy Distribution) fit to the PACS (Poglitsch et al. 2010) $160 \mu \mathrm{m}$, and the SPIRE (Griffin et al. 2010) 250, 350, and $500 \mu \mathrm{m}$ wavelengths observations. More details can be found in Appendix A and a full map of the column density of the Cygnus X North

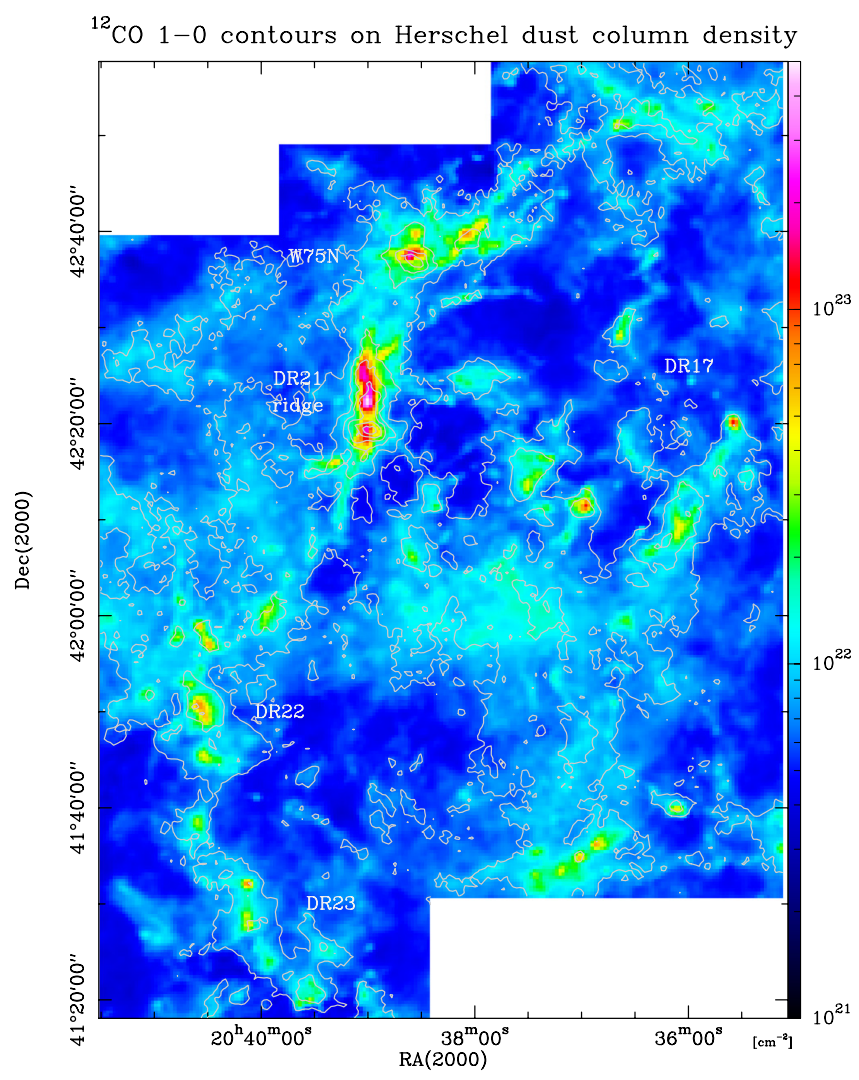

Fig. 1. ${ }^{12} \mathrm{CO} 1 \rightarrow 0$ contours of line integrated $\left(v=-10\right.$ to $20 \mathrm{~km} \mathrm{~s}^{-1}$ ) emission with levels 63.5 to $381 \mathrm{~K} \mathrm{~km} \mathrm{~s}^{-1}$ in steps of $63.5 \mathrm{~K} \mathrm{~km} \mathrm{~s}^{-1}$ overlaid on a Herschel dust column-density map at $36^{\prime \prime}$ resolution (that corresponds to $0.41 \mathrm{pc}$ at a distance of $1.4 \mathrm{kpc}$ ). This map was corrected for an average foreground contamination of $A_{v}=5$. Prominent features, such as $\mathrm{W} 75 \mathrm{~N}$ or the DR21 ridge, are indicated in the plot. DR17, 22, and 23 are $\mathrm{H}$ II regions.

region is shown in Schneider et al. (in prep.) and Bontemps et al. (in prep.). A smaller cutout of the DR21 ridge at 25" resolution is presented in Hennemann et al. (2012).

\section{The structure of the Cygnus $X$ North region}

Cygnus $\mathrm{X}$ is one of the richest star-formation regions in the Galaxy, containing the prominent OB-association Cyg OB2, with $~ 50$ O-stars (see Reipurth \& Schneider 2008 for a review). From large-scale ${ }^{13} \mathrm{CO} 2 \rightarrow 1$ (Schneider et al. 2006) and ${ }^{13} \mathrm{CO}$ $1 \rightarrow 0$ (Schneider et al. 2007) surveys, we derive a mass of a few $10^{6} M_{\odot}$ for the whole molecular cloud complex that is divided into the Cygnus X North and South regions. These studies show that the majority of the molecular clouds in the complex are located at a common distance of about 1.4-1.7 kpc. A distance of $1.4 \mathrm{kpc}$, which is derived from maser parallax (Rygl et al. 2012), is now commonly accepted. The clouds in the Cygnus $\mathrm{X}$ North region are actively forming stars and contain more than 100 massive pre- and protostellar dense cores (Motte et al. 2007; Bontemps 2010; Csengeri et al. 2011; Hennemann et al. 2012).

Figure 1 shows the dust column-density map of Cygnus $\mathrm{X}$ North with contours of ${ }^{12} \mathrm{CO} 1 \rightarrow 0$ overlaid onto it. The ${ }^{12} \mathrm{CO}$ emission is integrated over the velocity range -10 to $20 \mathrm{~km} \mathrm{~s}^{-1}$, which confines all star-forming molecular clouds that are directly associated with the Cyg OB2 cluster. Outside this velocity range, there are no other clouds along the line of sight, although in other regions of Cygnus $\mathrm{X}$, clouds from the Perseus 
N. Schneider et al.: Understanding star formation in molecular clouds. III.

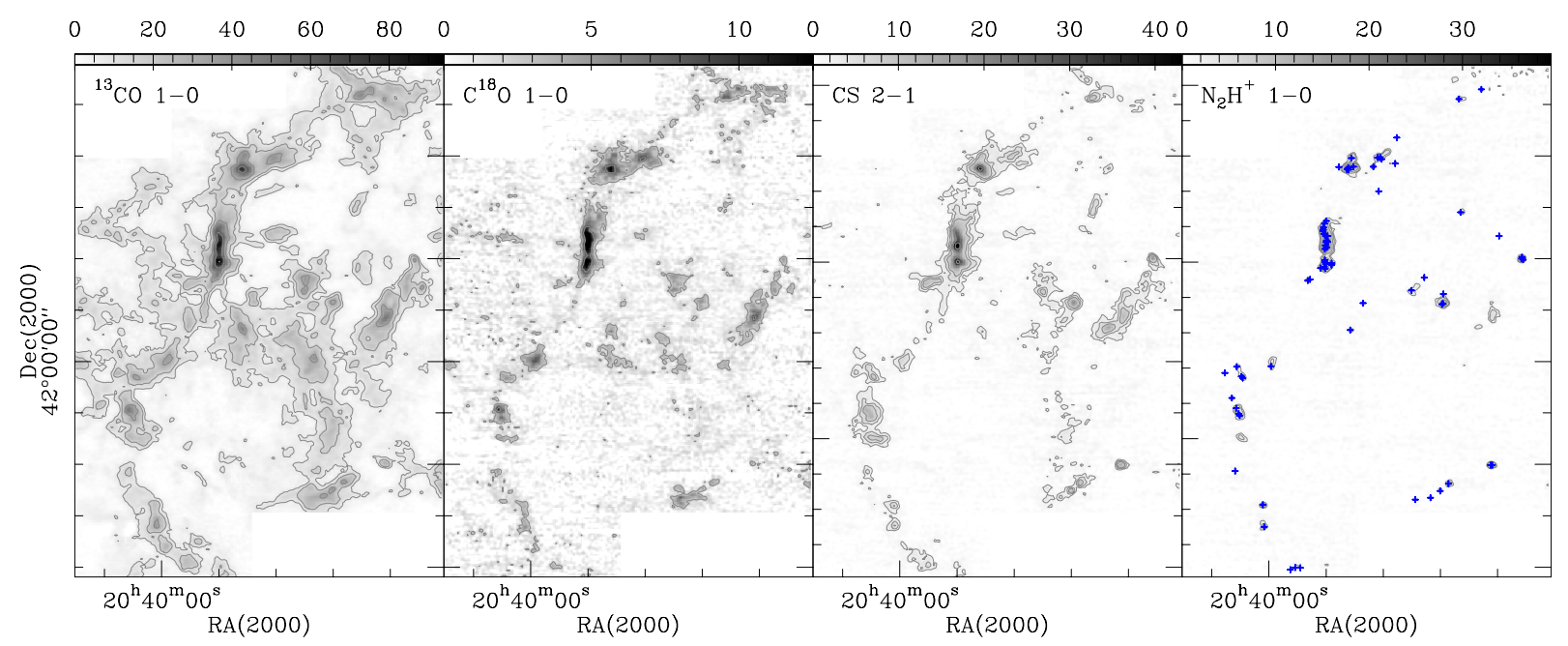

Fig. 2. Line integrated $\left(v=-10\right.$ to $20 \mathrm{~km} \mathrm{~s}^{-1}$ ) maps (in $\mathrm{K} \mathrm{km} \mathrm{s}^{-1}$ ) of molecular emission in Cygnus X North. The lower level is zero for all maps. The blue crosses in the $\mathrm{N}_{2} \mathrm{H}^{+}$map indicate submm-continuum sources (Motte et al. 2007).

arm appear at velocities around $-40 \mathrm{~km} \mathrm{~s}^{-1}$. As demonstrated in Schneider et al. (2006, see their Fig. 3), there are mainly two velocity coherent cloud complexes in Cygnus X North: the DR21 ridge-DR22-DR23 clouds at $v=-10$ to $1 \mathrm{~km} \mathrm{~s}^{-1}$ and the W75NDR17 clouds at $v=7$ to $20 \mathrm{~km} \mathrm{~s}^{-1}$. However, ${ }^{12} \mathrm{CO}$ and dust also trace more diffuse emission, mainly arising from the "Great Cygnus Rift", a nearby ( $0.6-1 \mathrm{kpc})$ region of obscuration, which is identified in optical images, and which is not associated with Cyg OB2. As shown in Schneider et al. (2007), the extinction due to the Rift is of the order of $A_{v} \sim 5-10$ and mainly arises between $\mathrm{v}=6$ to $20 \mathrm{~km} \mathrm{~s}^{-1}$. Other authors determine values between $A_{v}=2-5$ (Sale et al. 2009), $A_{v}=2.5-7$ (Drew et al. 2008), $A_{v}=5.5-7.5$ (Wright et al. 2010), and $A_{v}=2.6-5.6$ (Guarcello et al. 2012). An extinction around five is also the lowest emission level in the Herschel dust map that is analysed in this paper. The Rift emission is barely detected in ${ }^{13} \mathrm{CO}$ and not in $\mathrm{C}^{18} \mathrm{O}, \mathrm{CS}$, and $\mathrm{N}_{2} \mathrm{H}^{+}$. This means that we expect only the low column-density range of the ${ }^{12} \mathrm{CO}$ PDF to be affected. The higher column-density power-law tail is, in any case, not affected.

Figure 2 shows the same cutout of Cygnus X North as Fig. 1 in four different line tracers. The optical depth of the lines decreases from left to right and the critical density increases. While the CO $1 \rightarrow 0$ lines have a low critical density $n_{\text {cr }}$ of $\sim 10^{3} \mathrm{~cm}^{-3}$, the $\mathrm{CS} 2 \rightarrow 1$ and the $\mathrm{N}_{2} \mathrm{H}^{+} 1 \rightarrow 0$ lines have $n_{\mathrm{cr}}$ of $1.3 \times 10^{5} \mathrm{~cm}^{-3}$ and $6.1 \times 10^{4} \mathrm{~cm}^{-3}$, respectively, for a temperature of $10 \mathrm{~K}$ and thermal excitation (see. e.g. Flower 1999; Daniel et al. 2005; Shirley et al. 2015). In addition, $\mathrm{CO}$ depletes in cold and dense gas while CS, and in particular $\mathrm{N}_{2} \mathrm{H}^{+}$, remain stable in this gas phase. Accordingly, the maps show selectively different density regimes of the gas. While ${ }^{13} \mathrm{CO} 1 \rightarrow 0$ is still sensitive to lowerdensity gas and resembles the ${ }^{12} \mathrm{CO}$ map, $\mathrm{N}_{2} \mathrm{H}^{+}$is confined to the densest clumps. Basically all clumps visible in the $\mathrm{N}_{2} \mathrm{H}^{+}$ map contain cold, dense cores (starless and protostellar, indicated by crosses in Fig. 2) observed in mm-dust continuum with the MAMBO bolometer (Motte et al. 2007). From the submmdust observations, a typical size of $0.1 \mathrm{pc}(0.7 \mathrm{pc})$ and density of $10^{5} \mathrm{~cm}^{-3}\left(10^{4} \mathrm{~cm}^{-3}\right)$ for the cores (clumps) was derived. This value is confirmed by a decomposition of our $\mathrm{N}_{2} \mathrm{H}^{+}$map using the GAUSSCLUMPS algorithm (Stutzki \& Güsten 1990) in the velocity range -10 to $20 \mathrm{~km} \mathrm{~s}^{-1}$, which shows an average clump size of $\sim 0.6 \mathrm{pc}$. We only consider clumps larger than 1.5 times the beamsize $\left(45^{\prime \prime}\right)$.

\section{Probability distribution functions from dust and molecules}

\subsection{The dust PDF}

As discussed in the previous section, the dust map may suffer from foreground contamination that mainly arises from the Cygnus Rift. Contamination always modifies the measured cloud PDF. Relative to the underlying PDF, the measured PDF becomes narrower, the peak shifts towards higher $A_{v}$, and the slope of the power-law tail steepens (Schneider et al. 2015a). Following the method outlined in Schneider et al. (2015a), we can correct for foreground contamination by removing the constant $A_{v}=5$ value for the Cygnus Rift from the original columndensity map.

For a more realistic approach, we have to take into acount, however, that the foreground contamination will typically also show a distribution of column densities, usually also following a log-normal distribution. In a systematic study, Ossenkopf et al. (in prep.) show that the correction by a constant foreground subtraction still works quite well if the most probable contaminating column density is used as the offset. This is illustrated in Fig. 4. Following the description in Schneider et al. (2015a), we contaminate a model cloud with the parameters of the Cygnus PDF with a foreground cloud that is generated from a fractional Brownian motion (fBm) map. This has a power spectral index of 2.8, which matches the typically-observed spatial scaling behaviour, and has a lognormal column-density PDF with a width $\sigma_{\eta}=0.45$ and a contamination $A_{v}=5.0$. This cloud is thus a more realistic representation of a foreground or background contaminating cloud. Figure 4 shows that the contamination produces a distortion in the PDF but the simple correction obtained by subtracting a constant $A_{v}=5.0$ value fully recovers the PDF tail and recovers the PDF peak position approximately.

Even for such a relatively strong contamination, we can thus recover the main PDF properties by subtracting a constant offset for the contamination. Figure 3 shows the N-PDF obtained from Herschel dust continuum data, corrected for a contamination of $A_{v}=5$. This dust PDF shows the typical shape of PDFs obtained for star-forming regions: a lognormal distribution for low column densities followed by a power-law distribution between $A_{v} \sim 10-15$ and a few hundred $A_{v}$. Above $A_{v} \sim 100$, some excess in the PDF is observed that could constitute a second power law, when compared to a simple power law. Such an 


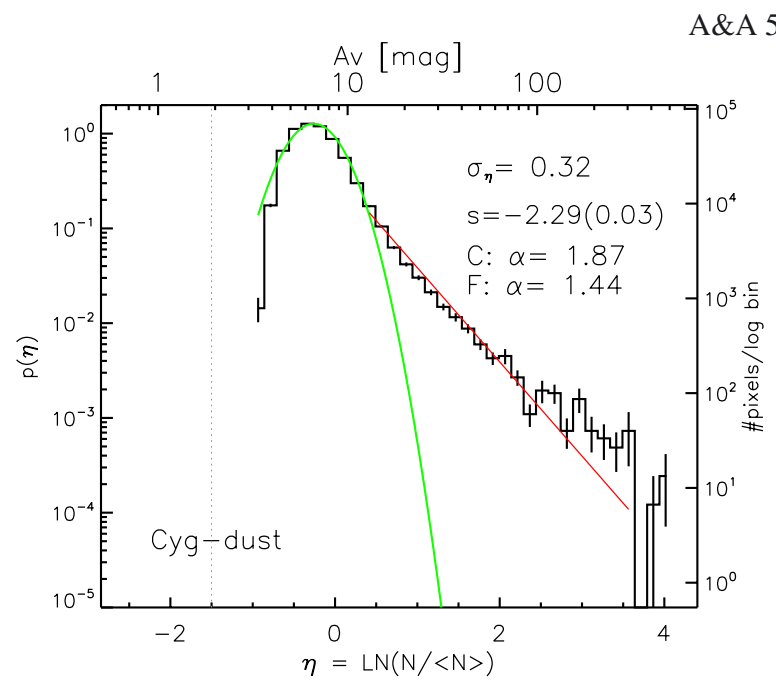

Fig. 3. PDF derived from the foreground-corrected Herschel columndensity map. The left $y$-axis gives the normalized probability density $p(\eta)$, the right $y$-axis the number of pixels per log bin. The upper $x$-axis is the visual extinction and the lower $x$-axis the natural logarithm of the normalized column density. The green curve indicates the best lognormal fit to the low column-density distribution, the red line displays a linear regression power-law fit to the high column-density tail. The dispersion of the fitted $\operatorname{PDF}\left(\sigma_{\eta}\right)$, the slope $s$ and its error, and the exponent $\alpha$ of an equivalent spherical density distribution (C) and a cylindrical density profile, such as for filaments $(\mathrm{F})$, are indicated.

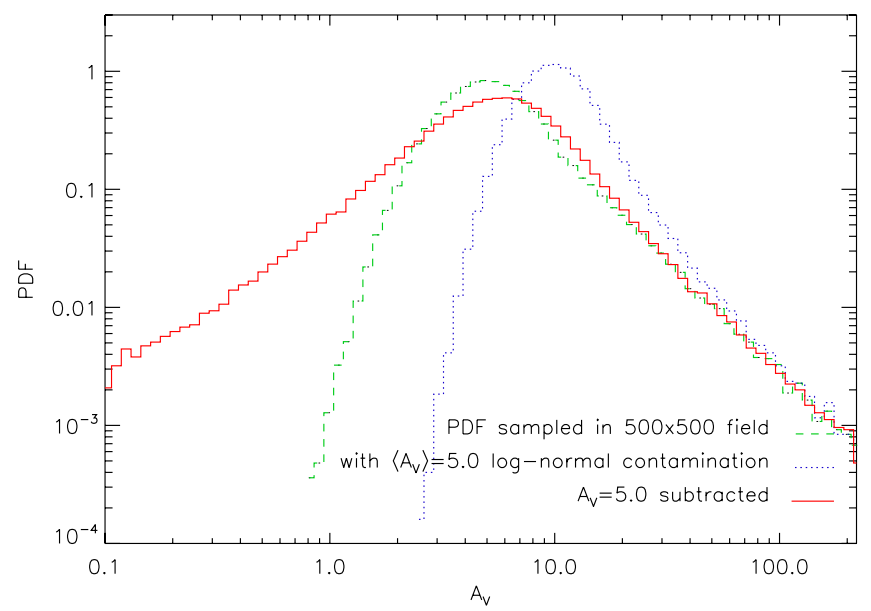

Fig. 4. Original PDF of a model cloud (see text) with a lognormal core and a power-law tail is shown with a green dashed line. This cloud is "contaminated" with a model cloud that has a lognormal core. The resulting PDF is shown as a blue dashed line. The red line then displays the "corrected" PDF if a constant value of $A_{v}=5$ is removed.

excess/flatter power-law tail was recently found in some massive GMCs (Schneider et al. 2015c) and was interpreted as a possible result of internal stellar feedback. The same argument may hold here because only pixels in the DR21 ridge, $\mathrm{W} 75 \mathrm{~N}$, and some small, isolated clumps - all associated with young stellar objects (YSOs) - contribute to this high column-density part of the PDF (see Fig. 1).

\subsection{The PDFs from $C O$}

The PDFs of $\mathrm{H}_{2}$ column densities $N_{\mathrm{mol}}\left(\mathrm{H}_{2}\right)$, which were obtained from molecular lines, are shown in Figs. 5 and 6, together with the same dust PDF from Fig. 3. Appendix B explains in detail how $N_{\text {mol }}\left(\mathrm{H}_{2}\right)$ was derived. In summary, we assume a

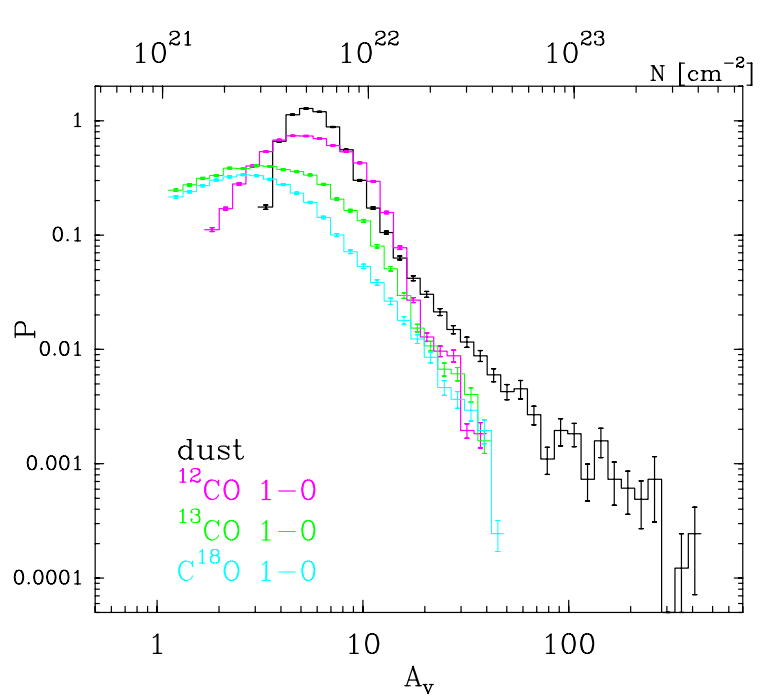

Fig. 5. Probability distribution functions of $\mathrm{H}_{2}$, obtained from dust and ${ }^{12} \mathrm{CO},{ }^{13} \mathrm{CO}$, and $\mathrm{C}^{18} \mathrm{O} 1 \rightarrow 0$. All PDFs were constructed using pixels above the $3 \sigma$ noise level.

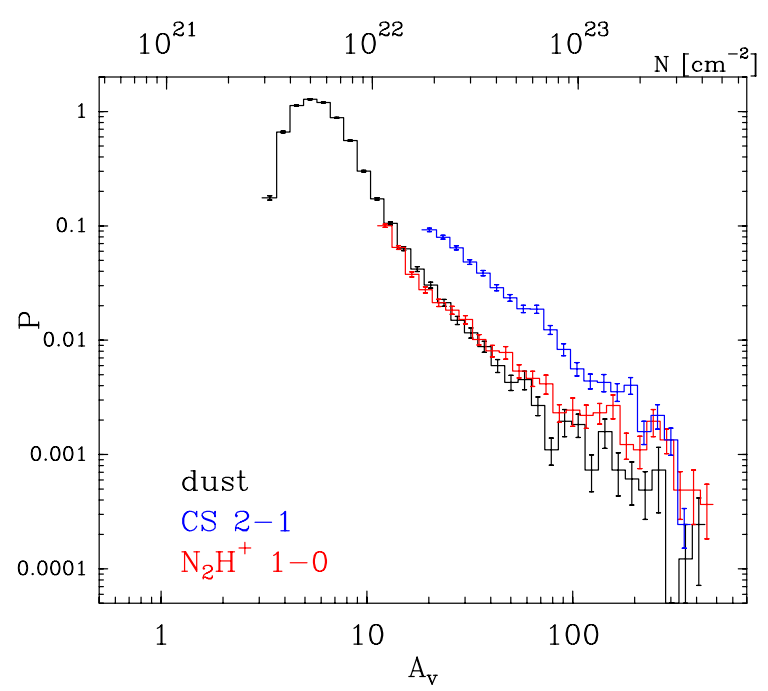

Fig. 6. Like Fig. 4 but showing the $\mathrm{CS} 2 \rightarrow 1$ and $\mathrm{N}_{2} \mathrm{H}^{+} 1 \rightarrow 0$ PDFs (converted into $\mathrm{H}_{2}$ column density) in addition to the dust PDF.

constant excitation temperature of $10 \mathrm{~K}$, LTE conditions, and a beam/source-filling factor of unity when determining all column densities. We note, however, that PDFs are always resolutionlimited. The PDFs were constructed from pixels above the $3 \sigma$ noise level and are normalized to the number of pixels from the dust map (\#pix dust $_{\text {) }}$, i.e. we scaled the molecular line PDFs by

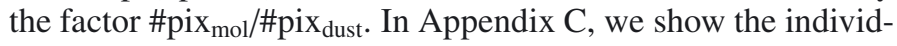
ual PDFs without this scaling. Above the noise level of $A_{v} \sim 1-2$, the $N_{\text {mol }}\left(\mathrm{H}_{2}\right)$-PDFs of ${ }^{12} \mathrm{CO},{ }^{13} \mathrm{CO}$, and $\mathrm{C}^{18} \mathrm{O}$ can be fitted by a broad lognormal distribution with a peak at $A_{v} \sim 3-5$, and by a power-law tail above $A_{v}=15$ for $\mathrm{C}^{18} \mathrm{O}$. Also, the ${ }^{13} \mathrm{CO} \mathrm{PDF}$ shows some excess above $A_{v} \sim 15$ (see Appendix C for a more detailed display).

Considering all uncertainties in the determination of $\mathrm{H}_{2}$ column density from dust and $\mathrm{CO}$, the lognormal part in the $\mathrm{CO}$ PDFs corresponds quite well to the lognormal part of the dust PDF, which indicates that the low-density molecular gas is probably well mixed with dust. The ${ }^{12} \mathrm{CO}$ PDF is a special case because it is contaminated by line-of-sight confusion from the 
Cygnus Rift at a distance of $600 \mathrm{pc}$, and thus shifts towards lower $A_{v}$ to some degree. For $A_{v}$ larger than $\sim 15$ (we note that the column densities are correct only within a factor $\sim 2$ ), however the ${ }^{12} \mathrm{CO}$ PDFs depart from the lognormal shape and the distribution becomes "bumpy" and falls off. This is the regime where the line no longer traces the dense gas because it becomes optically thick. In comparison to hydrodynamic simulations with radiative transfer (Shetty et al. 2011), our observed ${ }^{12}$ CO PDF covers a lower column-density range. Their "Milky Way cloud" (case (a) in Fig. 2) ${ }^{12} \mathrm{CO}$ PDF has the same width but peaks around $A_{v}=$ 10-15. More recent results of Szücs et al. (in prep.), however, show ${ }^{12}$ CO PDFs that cover a very similar column-density range to the one we observed.

The ${ }^{13} \mathrm{CO}$ and $\mathrm{C}^{18} \mathrm{O}$ PDFs extend to higher $A_{v}$ (up to $\sim 40$ ). The ${ }^{13} \mathrm{CO}$ line is optically thin $(\tau \sim 0.1-0.3$, see Fig. B.3) in most of the clouds and becomes marginally more optically thick $(\tau \sim 1)$ only in the DR21 ridge. The $\mathrm{C}^{18} \mathrm{O}$ line is optically thin everywhere with maximum values of $\tau \sim 0.4$ in the DR2 1 ridge. Optical depth effects, and not depletion, are the main reason for the different PDF shapes of ${ }^{12} \mathrm{CO},{ }^{13} \mathrm{CO}$, and $\mathrm{C}^{18} \mathrm{O}$ for higher column densities. The power-law behaviour for $A_{v}>15$ that we find for $\mathrm{C}^{18} \mathrm{O}$ indicates that the whole column of gas is traced up to $A_{v} \sim 40$. Because ${ }^{13} \mathrm{CO}$ becomes marginally optically thin, we do not observe a clear power-law tail but only some excess because the highest column densities are no longer traced. ${ }^{12} \mathrm{CO}$ becomes even more easily optically thick so that the high column-density pixels are not traced at all and the PDF appears lognormal. Depletion of all CO isotopologues probably sets in for $A_{v}$ larger than around 40 where all PDFs fall off. The removal of $\mathrm{CO}$ from the gas phase typically happens at densities above $10^{4} \mathrm{~cm}^{-3}$ at temperatures $\sim 15 \mathrm{~K}$ (e.g. Blake et al. 1995). Our value of $A_{v} \sim 40\left(N=3.8 \times 10^{22} \mathrm{~cm}^{-2}\right)$ thus corresponds to a typical clump size $d$ of $\sim 1.25 \mathrm{pc}\left(d=N /\left(10^{4} \mathrm{~cm}^{-3}\right)\right)$ in which $\mathrm{CO}$ depletes. These clumps are the ones that are well traced by $\mathrm{N}_{2} \mathrm{H}^{+}$emission and the temperature map (Fig. 8) that we discuss in the next section.

At the low $A_{v}$ end, approximately in the range $A_{v}=1-2$, we observe some excess compared to a purely lognormal PDF distribution for ${ }^{13} \mathrm{CO}$ and $\mathrm{C}^{18} \mathrm{O}$ (Fig. C.1). The chemistry in this column-density regime is governed by $(\mathrm{F}) \mathrm{UV}$ radiation via photodissociation $\left(A_{v}<5\right)$ and fractionation $\left(A_{v}<3\right)$ processes (e.g. van Dishoeck \& Black 1988). The excess indicates that selfshielding of ${ }^{13} \mathrm{CO}$ and $\mathrm{C}^{18} \mathrm{O}$ against UV-radiation is rather effective. Otherwise, the abundances would be lower and the PDF would drop more significantly. On the other hand, in warmer regions (internally or externally heated) in the medium $A_{v}$ range, $\mathrm{CO}$ desorption leads to a higher abundance of $\mathrm{CO}$. These spatial abundance variations, which were observed for ${ }^{13} \mathrm{CO}$ in Perseus (Pineda et al. 2008) and Orion (Ripple et al. 2013), have an obvious influence on the N-PDF. However, they are difficult to assess (see next section).

\subsection{The PDFs from $\mathrm{CS}$ and $\mathrm{N}_{2} \mathrm{H}^{+}$}

The $N_{\text {mol }}\left(\mathrm{H}_{2}\right)$-PDFs of $\mathrm{CS}$ and $\mathrm{N}_{2} \mathrm{H}^{+}$mostly cover the high column-density range and can be best fitted by a pure power law (see Appendix C). However, all absolute values need to be taken with caution because they strongly depend on the abundance values that were used for converting column densities into $\mathrm{H}_{2}$ and on the excitation temperature.

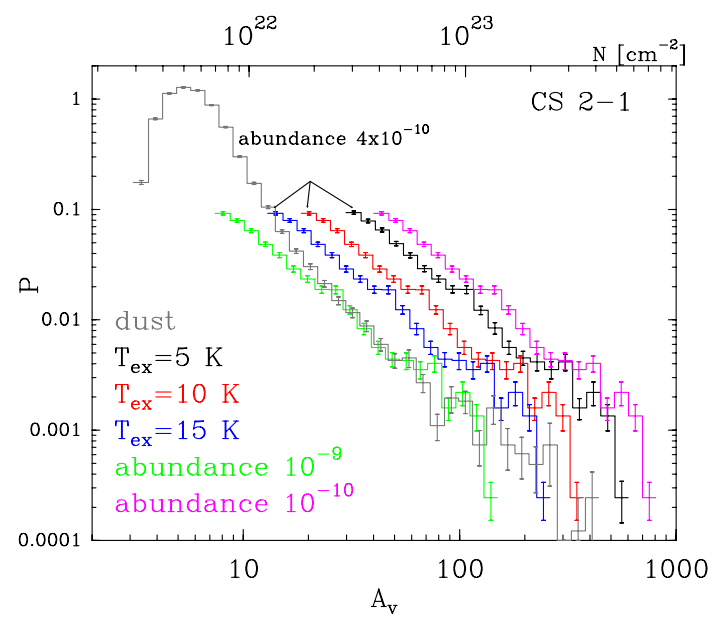

Fig. 7. N-Probability distribution functions obtained from CS using (i) different excitation temperatures $(5,10,15 \mathrm{~K})$ and an abundance of $4 \times 10^{-10}$; and (ii) at a temperature of $10 \mathrm{~K}$ but abundance values of $10^{-10}$ and $10^{-9}$. The dust PDF is shown for comparison.

For $\mathrm{N}_{2} \mathrm{H}^{+}$, we adopt $\left[\mathrm{N}_{2} \mathrm{H}^{+}\right] /\left[\mathrm{H}_{2}\right]=5 \times 10^{-10}$, a value determined for massive ${ }^{3}$ cloud cores (Pigorov et al. 2003), and applied it to one of the Cygnus X North cores (N63, Motte et al. 2007) in the following way (Fechtenbaum et al., in prep.): Level population diagrams that use lines of the optically thin isotopologues ${ }^{15} \mathrm{~N}_{2} \mathrm{H}^{+}$and $\mathrm{N}^{15} \mathrm{NH}^{+}$were plotted to estimate their column density. An isotopic ratio ${ }^{14} \mathrm{~N} /{ }^{15} \mathrm{~N}$ of 448 (Wilson \& Rood 1994) was used to derive the column density of $\mathrm{N}_{2} \mathrm{H}^{+}$. The mass of $44 M_{\odot}$ in a 30" beam (Duarte-Cabral et al. 2013) of the source enabled us to calculate the column density of $\mathrm{H}_{2}$ and thus the abundance of $\mathrm{N}_{2} \mathrm{H}^{+}$. The resulting PDF covers a column-density regime of $A_{v} \sim 15$ to 400 , which corresponds very well to the one derived from dust (see Fig. 6).

For CS, there is a large scatter in observed values for highmass star-forming regions with $[\mathrm{CS}] /\left[\mathrm{H}_{2}\right]=10^{-9}$ to a few times $10^{-10}$ (Gianetti et al. 2012; Li et al. 2015; Neufeld et al. 2015). Low-mass cores have typical values of a few times $10^{-9}$ (e.g. Tafalla et al. 2006). Here, we use a value of $4 \times 10^{-10}$ (Fechtenbaum et al., in prep.) that was obtained using the optically thin isotopologue $\mathrm{C}^{33} \mathrm{~S}$ and applying the same method as outlined above. The PDF obtained with this abundance value covers the $A_{v}$ range $\sim 20$ to 350 and thus has a similar column density range as the PDF determined from dust, but shifted vertically to the latter.

To investigate in more detail the effects of different excitation temperatures and abundances, in Fig. 7 we plot a whole set of PDFs for CS. We first vary the excitation temperature, using 5 and $15 \mathrm{~K}$ as two extreme cases, and $10 \mathrm{~K}$ (average value determined from all molecular lines and dust). The column density variation is significant, increases its value by more than a factor two between $T_{\mathrm{ex}}=15 \mathrm{~K}$ and $5 \mathrm{~K}$. Different abundance values obviously have a significant impact on the PDF as well. A lower (but unlikely) $[\mathrm{CS}] /\left[\mathrm{H}_{2}\right]$ ratio of $10^{-10}$ shifts the PDF to higher column densities with unrealistic values of a few hundred $A_{v}$. A higher abundance shifts the PDF towards lower column densities, and a value of $10^{-9}$ leads to a PDF that corresponds to the one obtained from dust. In this way, it is possible to "calibrate" the $[\mathrm{CS}] /\left[\mathrm{H}_{2}\right]$ abundance using the dust PDF. However, this method should be treated with caution because the

3 Lower abundances around 1-3 $\times 10^{-10}$ were found in low-mass dense cores and dark clouds (e.g. Caselli et al. 2002; Bergin et al. 2001; Tafalla et al. 2006). 

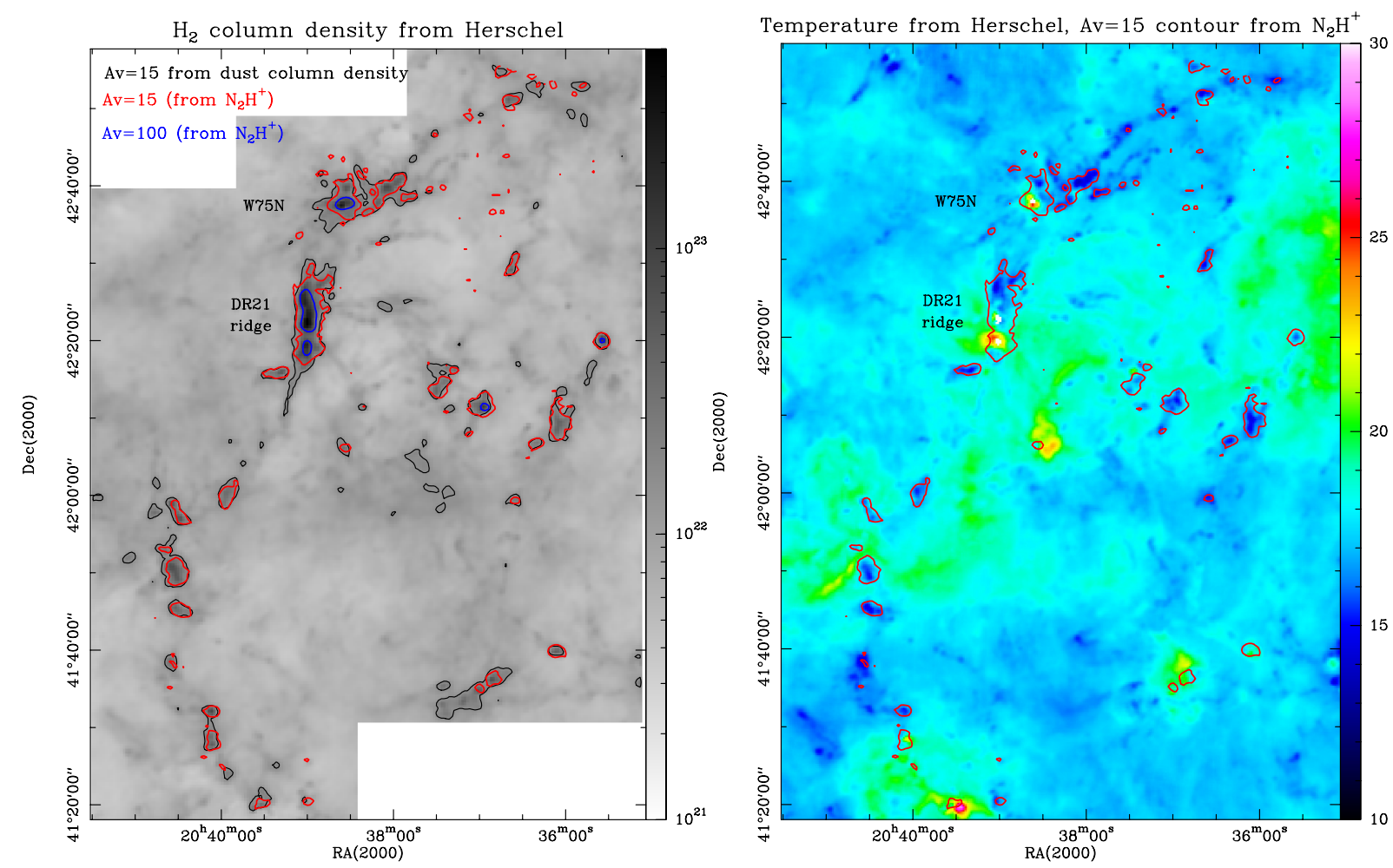

Fig. 8. Left: foreground-corrected dust column-density map (as shown in Fig. 1) in greyscale. The black contour indicates the $A_{v}=15$ level. The red contours show the same $A_{v}=15$ level from the $\mathrm{H}_{2}$ column-density map derived from $\mathrm{N}_{2} \mathrm{H}^{+}$, using an excitation temperature of $10 \mathrm{~K}$ and an abundance of $2 \times 10^{-9}$. Right: dust temperature map of Cygnus X North from Herschel with the same red $A_{v}=15$ contour from $\mathrm{N}_{2} \mathrm{H}^{+}$.

molecular line PDF also depends on the excitation temperature, and the dust PDF has its own uncertainties (variable temperature along the line of sight, unknown dust opacity etc.).

\subsection{The high column density range in the maps}

The PDFs obtained from dust emission and $\mathrm{N}_{2} \mathrm{H}^{+}$(and CS) both show a power-law tail starting around $A_{v} \sim 15$. Figure 8 (left panel) illustrates the high spatial correspondence between the pixels in the dust and $\mathrm{N}_{2} \mathrm{H}^{+}$maps that cover the same columndensity range. The black and red contours indicate the $A_{v}=15$ level for the $\mathrm{H}_{2}$ column density derived from dust and $\mathrm{N}_{2} \mathrm{H}^{+}$, respectively. On average, both contours cover the same areas, implying that the dust is well mixed with the dense and cold gas that is traced by $\mathrm{N}_{2} \mathrm{H}^{+}$. The geometry of the emitting regions is a mixture between spherical and filamentary. Even at very high column densities (indicated by the arbitrary chosen $A_{v}=100$ blue contour from $\mathrm{N}_{2} \mathrm{H}^{+}$), not only spherical clumps are outlined but also the elongated DR21 ridge. An overlay between the dust temperature obtained with Herschel and the same $A_{v}=15$ contour from $\mathrm{N}_{2} \mathrm{H}^{+}$(Fig. 8, right panel) shows that it is indeed mostly cold gas traced by $\mathrm{N}_{2} \mathrm{H}^{+}$and dust. The clumps that are indicated by the red contour typically have a temperature of 10-15 K, except those with internal sources (the DR21 region in the DR21 ridge and DR23 at the southern border of the map). In these cases, the column density is underestimated so that the true power-law tails of both, the dust, and $\mathrm{N}_{2} \mathrm{H}^{+} \mathrm{PDF}$, which is shown in Fig. 6, are slightly flatter.

\section{Discussion and conclusions}

In the following section, we base our discussion on the column density maps and PDFs of $\mathrm{CS}$ and $\mathrm{N}_{2} \mathrm{H}^{+}$derived using
Table 1. Results from the power-law fit to the PDFs.

\begin{tabular}{lcccccc}
\hline \hline Species & $s$ & $\Delta s$ & $\alpha_{\mathrm{c}}$ & $\Delta \alpha_{\mathrm{c}}$ & $\alpha_{\mathrm{f}}$ & $\Delta \alpha_{\mathrm{f}}$ \\
\hline Dust & -2.29 & 0.03 & 1.87 & 0.03 & 1.44 & 0.03 \\
$\mathrm{C}^{18} \mathrm{O} 1 \rightarrow 0$ & -2.57 & 0.12 & 1.78 & 0.04 & 1.39 & 0.04 \\
$\mathrm{CS} 2 \rightarrow 1$ & -1.56 & 0.02 & 2.28 & 0.02 & 1.64 & 0.02 \\
$\mathrm{~N}_{2} \mathrm{H}^{+} 1 \rightarrow 0$ & -1.41 & 0.03 & 2.42 & 0.03 & 1.71 & 0.03 \\
\hline
\end{tabular}

Notes. $s$ and $\Delta s$ are the slope and its error, $\alpha_{\mathrm{c}}$ and $\alpha_{\mathrm{f}}$ are the exponents of a density distribution with $\rho \propto r^{-\alpha}$ with spherical and filamentary geometry.

an excitation temperature of $10 \mathrm{~K}$ and abundance values of $[\mathrm{CS}] /\left[\mathrm{H}_{2}\right]=10^{-9}$ (to be consistent with the dust PDF) and $\left[\mathrm{N}_{2} \mathrm{H}^{+}\right] /\left[\mathrm{H}_{2}\right]=5 \times 10^{-10}$, respectively.

\subsection{Thermal and subthermal excitation regimes}

For a homogeneous medium with beam-filling of unity, the $\mathrm{H}_{2}$ column-density threshold $N$ for thermally excited $\mathrm{N}_{2} \mathrm{H}^{+}$and CS calculates from the clump size $(d)$ and critical densities with $N=$ $d \times n_{\mathrm{cr}}$. For $d$ we adopt $0.7 \mathrm{pc}$, taken from dust submm observations (Motte et al. 2007). This value is close to the average clump size of $0.6 \mathrm{pc}$ we derive from our $\mathrm{N}_{2} \mathrm{H}^{+}$clump decomposition ${ }^{4}$. We thus obtain $A_{v}=285$ and 130 for $\mathrm{CS}$ and $\mathrm{N}_{2} \mathrm{H}^{+}$, respectively, assuming critical densities of $1.3 \times 10^{5} \mathrm{~cm}^{-3}\left(6.1 \times 10^{4} \mathrm{~cm}^{-3}\right)$ for $\mathrm{CS}\left(\mathrm{N}_{2} \mathrm{H}^{+}\right)$. Above these values, the emission lines should

\footnotetext{
4 The submm continuum observations have an angular resolution of $11^{\prime \prime}$, compared to the $45^{\prime \prime}$ for $\mathrm{N}_{2} \mathrm{H}^{+}$, and trace a much larger column density regime. They are thus more precise for separating individual clumps.
} 
be thermalized. However, the clumpiness of the gas leads to a lower threshold of thermalization. With an average density $\langle n\rangle=10^{4} \mathrm{~cm}^{-3}$ of the clumps, which was determined from submm continuum observations (Motte et al. 2007), we obtain a beam filling $\left(\langle n\rangle / n_{\mathrm{cr}}\right)$ of $\sim 10 \%$ for CS and $\sim 15 \%$ for $\mathrm{N}_{2} \mathrm{H}^{+}$. Both lines are thus already thermalized at lower $A_{v}$. For $\mathrm{N}_{2} \mathrm{H}^{+}$ we independently derive its thermal excitation by the hyperfinestructure line ratios. The line is mostly thermally excited because we obtain excitation temperatures above $5 \mathrm{~K}$ for the emitting gas (Appendix C), which is above the typical value for subthermal excitation.

CS has a higher critical density but depletes at densities above $\sim 10^{5} \mathrm{~cm}^{-3}$, which is consistent with our PDF that is cut off at $A_{v} \sim 100$ (for the abundance $[\mathrm{CS}] /\left[\mathrm{H}_{2}\right]=10^{-9}$ ). On the other hand, its chemistry is less density dependent. Hence, we expect that CS is not thermalized at the lowest end of the distribution.

\subsection{Slopes of the N-PDFs}

Independent of the exact column-density regime that is covered by the PDFs from dust and $\mathrm{CS} / \mathrm{N}_{2} \mathrm{H}^{+}$, they all show power-law tails with slopes $s$ between -1.4 and -2.3 (see Table 1). The slope $s$ of the power law can be converted into the exponent $\alpha$ of an equivalent density distribution $\rho \propto r^{-\alpha}$ (Appendix D). For spherical geometry, representing a single core or a core ensemble (Girichidis et al. 2014), we use $\alpha_{\mathrm{c}}=1-2 / \mathrm{s}$ (Federrath $\&$ Klessen 2013). For singular polytropic cylinders, which portray filaments, we use $\alpha_{\mathrm{f}}=1-1 / s$ (see Toci \& Galli (2015) for regular polytropic cylinder models and Fischera (2014) and Myers (2015) for the corresponding N-PDFs). The values we obtain for $\alpha\left(\alpha_{\mathrm{c}}\right.$ or $\left.\alpha_{\mathrm{f}}\right)$ vary between 1.4 and 2.4. In the simplified picture of the free-fall of a collapsing sphere, $\alpha=2$ for early stages and $\alpha=1.5$ after a singularity formed at the centre of the sphere (Shu 1977; Larson 1969; Penston 1969; Whitworth $\&$ Summers 1985). Our observed values from the dust $\left(\alpha_{\mathrm{c}}=1.9\right)$ and $\mathrm{C}^{18} \mathrm{O}\left(\alpha_{\mathrm{c}}=1.8\right)$ PDF slopes are consistent with the spherical free-fall scenario, but CS and $\mathrm{N}_{2} \mathrm{H}^{+}$have higher values with $\alpha_{\mathrm{c}}=2.3$ and $\alpha_{\mathrm{c}}=2.4$, respectively. All high-density clumps traced in CS and $\mathrm{N}_{2} \mathrm{H}^{+}$emission are associated with pre- and protostellar sources (Motte et al. 2007), which indicates that gravitational collapse in the star-forming phase has started. The high column densities seen in dust, $\mathrm{CS}$, and $\mathrm{N}_{2} \mathrm{H}^{+}$are thus not a consequence of a long column of diffuse emission, but correspond to spatially concentrated high volume densities.

On the other hand, the purely spherical free-fall picture seems unlikely to apply to most of the gas which defines the $\mathrm{N}-\mathrm{PDF}$ power laws, because most of the gas we observe in Cygnus $\mathrm{X}$ is filamentary. This is clearly illustrated in the maps shown in Figs. 1, 2, and 7, which display the filamentary network in the $\mathrm{CO}$ lines and, with only a small fraction of gas in cores, that could be considered roughly spherical (see CS and $\mathrm{N}_{2} \mathrm{H}^{+}$maps in Figs. 2 and 7 and submm continuum observations in Motte et al. 2007) ${ }^{5}$. Filaments can be in global free-fall collapse, as suggested by molecular line observations (e.g. Peretto et al. 2013; Schneider et al. 2010, 2015b) but the gas we see in filaments (and cores) can account for power-law $\mathrm{N}-\mathrm{PDF}$ if it has the right power-law radial-density structure, even in hydrostatic balance with no net inward motion. The observed N-PDF slopes ( $s=-1.4$ to -2.6 , leading to $\alpha_{\mathrm{f}}$ between 1.4 and 1.7) are consistent with self-gravitating but noncollapsing filament models (Myers 2015; Toci \& Galli 2015). In

\footnotetext{
In Aquila (Könyves et al. 2015) the power-law tail contains more than $\sim 50 \%$ of the mass in filaments but only $\sim 15 \%$ in cores.
}

this case, filamentary gas that has a sufficient mass per length ratio is centrally concentrated and self-gravitating, but does not need to be globally collapsing. However, to confine the filaments/clumps, an external pressure $p_{\text {ext }} \propto\langle n\rangle T_{\text {kin }}$ of the order of $p_{\text {ext }} \sim 10^{4-5} \mathrm{~cm}^{-3} \times 10 \mathrm{~K}$ is required (where $\langle n\rangle$ is the average density in the filaments/clumps and $T_{k i n}$ the gas temperature). However, this pressure cannot be isotropic, provided by the lower density envelope that is well traced in ${ }^{12} \mathrm{CO}$ at a density of $\sim 10^{3} \mathrm{~cm}^{-3}$ and a temperature of $\sim 10-40 \mathrm{~K}$. Moreover, it could be ram pressure of new gas that is accreting onto filaments and clumps, a process that is now frequently observed (e.g. Schneider et al. 2010; Kirk et al. 2013; Palmeirim et al. 2013).

The flatter power-law slopes of the CS and $\mathrm{N}_{2} \mathrm{H}^{+}$PDF in comparison to the dust could reflect an abundance change because $\mathrm{N}_{2} \mathrm{H}^{+}$is produced when $\mathrm{CO}$ is depleted at very high column densities (Bergin et al. 2001; Tafalla et al. 2002, 2002). In addition, because CS is not thermalized at the lowest column densities, this could lead to a somewhat flatter tail with respect to the dust.

In summary, a power-law distribution in the density structure is required to have a power-law tail in the PDF and this can be achieved by either a hydrostatic configuration, where the power law arises from a balance of gravitational forces and pressure gradients, or directly in a dynamically collapsing system. Probably both play a role, with filaments possibly balanced or at least contraction-slowed by (magnetic) pressure gradients on the one hand and very dense collapsing cores on the other.

\section{Summary}

We derived probability distribution functions (N-PDFs) of $\mathrm{H}_{2}$ column density for the Cygnus $\mathrm{X}$ North region from dust, ${ }^{12} \mathrm{CO}$, ${ }^{13} \mathrm{CO}, \mathrm{C}^{18} \mathrm{O} 1 \rightarrow 0, \mathrm{CS} 2 \rightarrow 1$, and $\mathrm{N}_{2} \mathrm{H}^{+} 1 \rightarrow 0$. The determination of the $\mathrm{H}_{2}$ column density from the molecular lines is based on standard procedures and abundances, assuming a common excitation temperature of $10 \mathrm{~K}$ and LTE. Our findings are:

- The PDFs of dust and CO isotopologues can be described by a lognormal distribution for the low column-density range between $A_{v} \sim 1$ to $\sim 15$, with a common peak around $A_{v} \sim 5$. Though line-of-sight contamination and variations in abundance and excitation temperatures introduce large uncertainties in the CO-PDFs, the overall correspondence between these observational PDFs and the ones from recent simulations (Szücs et al. 2015) is good.

- Optical depth effects are the main reason for the different $\mathrm{PDF}$ shapes of ${ }^{12} \mathrm{CO},{ }^{13} \mathrm{CO}$, and $\mathrm{C}^{18} \mathrm{O}$ for higher column densities. The PDF from ${ }^{12} \mathrm{CO}$ is cut above $A_{v} \sim 30$ because the line becomes optically thick. The marginally optically thin ${ }^{13} \mathrm{CO}$ line shows excess in its PDF for $A_{v}=15-40$, while the PDF for the optically thin $\mathrm{C}^{18} \mathrm{O}$ line displays a power-law tail in the same $A_{v}=15-40$ range.

- Neither selective photodissociation nor fractionation seem to play a significant role for the $\mathrm{CO}$ abundances for low column densities.

- Depletion of all CO isotopologues probably sets in for $A_{v}$ greater than around 40 where all PDFs fall off.

- The PDFs of CS and $\mathrm{N}_{2} \mathrm{H}^{+}$only consist of a power-law tail that covers a high column density range $\left(A_{v} \sim 15\right.$ to a few $100 A_{v}$ ). Using CS as an example, we discuss the potential influence of abundance and excitation temperature variations, and show that these could shift the entire PDF by more than one magnitude in column density. To better constrain this 
uncertainty, we "calibrate" the molecular line PDF using the dust-emission map (i.e. shifting the distribution at a given excitation temperature so that it corresponds to the dust PDF). For CS, we obtain an abundance of $[\mathrm{CS}] /\left[\mathrm{H}_{2}\right]=10^{-9}$ in this way.

- We find that the $\mathrm{N}_{2} \mathrm{H}^{+}$and CS lines are mainly thermally excited. $\mathrm{N}_{2} \mathrm{H}^{+}$is well mixed with the dust and traces spatially the same dense gas clumps of a typical size of $\sim 0.6-0.7$ pc that were shown to contain pre- and protostellar sources (Motte et al. 2007).

- The slopes of the high column-density power-law tail end of the N-PDFs from dust, CS, and $\mathrm{N}_{2} \mathrm{H}^{+}$are $s=-2.3,-1.6$, and -1.4 , respectively. These values are consistent with a gas distribution that is dominated by gravity, i.e. free-falling gas in cores and filaments, although a hydrostatic configuration with ram pressure by gas accretion can also take place. The power law then arises from a balance of gravitational forces and pressure gradients.

Summarising the observational results, we find that the N-PDFs obtained from molecular lines are not well confined because they depend strongly on excitation temperature and abundance, and various combinations of these can lead to the same PDF. "Calibrating" a molecular line PDF with dust is an appealing approach but it should be kept in mind that dust PDFs also suffer from uncertainties. For example, the specific dust opacity is not constant (a value of $\beta=2$ was chosen for this paper) and line-ofsight contamination can lead to an overestimation of the column density. However, this study nevertheless shows that the dust provides the highest dynamic range in tracing the low to high column-density regime, compared to the molecular line tracers (see also Goodman et al. 2009; and Burkhart et al. 2013).

Acknowledgements. N.S. and S.B. acknowledge support by the ANR-11-BS56010 project STARFICH. N.S., V.O., and T.C. acknowledge support from the Deutsche Forschungsgemeinschaft, DFG, through project number 0s 177/21 and 177/2-2, and central funds of the DFG-priority program 1573 (ISMSPP). C.F. acknowledges funding provided by the Australian Research Councils Discovery Projects (grants DP130102078 and DP150104329). R.S.K. acknowledges subsidies from the DFG priority program 1573 (Physics of the Interstellar Medium) and the collaborative research project SFB 881 (The Milky Way System, subprojects B1, B2, and B5).

\section{References}

Alves de Oliveira, C., Schneider, N., Merin, B., et al. 2014, A\&A, 568, A98 Bergin, E. A., Ciardi, D. R., \& Lada, C. 2001, ApJ, 557, 209

Blake, G. A., Sandell, G., van Dishoeck, E. F., et al. 1995, ApJ, 441, 689

Bohlin, R. C., Savage, B. D., \& Drake, J. F. 1978, ApJ, 224, 132

Bontemps, S., Motte, F., Csengeri, T., et al. 2010, A\&A, 524, A18

Bolatto, A. D., Wolfire, M., \& Leroy, A. K. 2013, ARA\&A, 51, 207

Burkhart, B., Ossenkopf, V., Lazarian, A., \& Stutzki, J. 2013, ApJ, 771, 122

Carlhoff, P., Schilke, P., Nguyen-Luong, Q., et al. 2013, A\&A, 560, A24

Caselli, P., Benson, P. J. Myers, P. C., \& Tafalla, M. 2002, ApJ, 572, 238

Csengeri, T., Bontemps, S., Schneider, N., et al. 2011, A\&A, 527, A135

Daniel, F., Dubernet, M.-L., Meuwly, M., et al. 2005, MNRAS, 363, 1083

Drew, J. E., Greimel, R., Irwin, M. J., \& Sale, S. E. 2008, MNRAS, 386, 1761

Duarte-Cabral, A., Bontemps, S., Motte, F., et al. 2013, A\&A, 558, A125

Federrath, C., \& Klessen, R. S. 2012, ApJ, 761, 156

Federrath, C., \& Klessen, R. S. 2013, ApJ, 763, 51

Federrath, C., Klessen, R. S., \& Schmidt, W. 2008, ApJ, 688, L79

Federrath, C., Roman-Duval, J., Klessen, R. J., et al. 2010, A\&A, 512, A81

Fischera, J. 2014, A\&A, 571, A95
Flower, D. R. 1999, MNRAS, 305, 651

Fontani, F., Giannetti, A., Beltran, M. T., et al. 2012, MNRAS, 423, 2342

Frerking, M. A., Langer, W. D., \& Wilson, R. W. 1982, ApJ, 262, 590

Froebrich, D., \& Rowles, J. 2010, MNRAS, 406, 1350

Gianetti, A., Brand, J., Massi, F., et al. 2012, A\&A, 538, A41

Girichidis, P., Konstandin, L., Whitworth, A. P., Klessen, R., et al. 2014, ApJ 781, 91

Goldsmith, P. F., Heyer, M., Narayanan, G., et al. 2008, ApJ, 680, 428

Goodman, A. A., Pineda, J. E., \& Schnee, S. L. 2009, ApJ, 692, 91

Griffin, M., Abergel, A., Abreau, S., et al. 2010, A\&A, 518, L3

Guarcello, M. G., Wright, N. J., Drake, J. J., et al. 2012, ApJS, 202, 19

Hennebelle, P., \& Chabrier, G. 2008, ApJ, 684, 395

Hennemann, M., Motte, F., Schneider, N., et al. 2012, A\&A, 543, L3

Hill, T., Motte F., Didelon P., et al. 2011, A\&A, 533, A94

Hopkins, P. F. 2013, MNRAS, 423, 2037

Kainulainen, J., Beuther, H., Henning, T., \& Plume, R. 2009, A\&A, 508, L35

Kirk, H., Myers, P. C., Bourke, T. L., et al. 2013, ApJ, 766, 115

Klessen, R. S. 2000, ApJ, 535, 869

Könyves, V., André P., Men'shchikov A., et al. 2015, A\&A, 584, A91

Krumholz, M., \& McKee, C. F. 2005, ApJ, 630, 250

Langer, W. D., \& Penzias, A. A. 1990, ApJ, 357, 477

Larson, R. B. 1969, MNRAS, 145, 271

Li, J., Wang, J., Zhu, Q., et al. 2015, ApJ, 802, 40

Lo, N., Cunningham, M. R., Jones, P. A., et al. 2009, MNRAS, 395, 1021

Lombardi, M., Lada, C., \& Alves, J. 2008, A\&A, 489, 143

Mangum, J. G., \& Shirley, Y. L. 2015, PASP, 127, 266

Motte, F., Bontemps, S., Schilke, P., et al. 2007, A\&A, 476, 1243

Motte, F., Zavagno A., Bontemps, S., et al. 2010, A\&A, 518, L77

Myers, P. C. 2015, ApJ, 806, 226

Neufeld, D. A., Godard, B., Gerin, M., et al. 2015, A\&A, 577, A49

Padoan, P., \& Nordlund, A. 2011, ApJ, 741, 22

Palmeirirm, P., André P., Kirk, J., et al. 2013, A\&A, 550, A38

Penston, M. V. 1969, MNRAS, 144, 425

Peretto, N., Fuller, G. A., Duarte-Cabral, A., et al. 2013, A\&A, 555, A112

Pineda, J. E., Caselli, P., \& Goodman, A. 2008, ApJ, 679, 481

Pineda, J. L., Goldsmith, P. F., Chapman N., et al. 2010, ApJ, 721, 686

Pigorov, L., Zinchenko, I., Caselli, P., et al. 2003, A\&A, 405, 639

Poglitsch, A., Waelkens, C., Geis, N., et al. 2010, A\&A, 518, L2

Reipurth, B., Schneider, N. 2008, Handbook of star forming regions, Vol. I, ASP Publ., ed. B. Reipurth, 36

Ripple, F., Heyer, M. H., Gutermuth, R., et al. 2013, MNRAS, 431, 1296

Russeil, D., Schneider, N., Anderson, L., et al. 2013, A\&A, 554, A42

Rygl, K. L. J., Brunthaler, A., Sanna, A., et al. 2012, A\&A, 539, A79

Sale, S. E., Drew, J. E., Unruh, Y. C., et al. 2009, MNRAS, 392, 497

Schneider, N., Bontemps, S., Simon, R., et al. 2006, A\&A, 458, 855

Schneider, N., Simon, R., Bontemps, S, et al. 2007, A\&A, 474, 873

Schneider, N., Csengeri T., Bontemps S., et al. 2010, A\&A, 520, A49

Schneider, N., Bontemps, S., Simon, R., et al. 2011, A\&A, 529, L1

Schneider, N., Csengeri, T., Hennemann, M., et al. 2012, A\&A, 540, L11

Schneider, N., André, Ph., Könyves, V., Bontemps, S., et al. 2013, ApJ, 766, L17

Schneider, N., Ossenkopf, V., Csengeri, T., et al. 2015a, A\&A, 575, A79

Schneider, N., Csengeri, T., Klessen, R. S., et al. 2015b, A\&A, 578, A29

Schneider, N., Bontemps, S., Girichidis, P., et al. 2015c, MNRAS, 453, L41

Shirley, Y. L. 2015, PASP, 127, 299

Shetty, R., Glover, S. C., Dullemond, C. P., \& Klessen, R. S. 2011, MNRAS, 412, 1686

Shu., F. 1977, ApJ, 214, 488

Strong, J., Bloemen, J., Dame, T., et al. 1988, A\&A, 207, 1

Stutzki, J., \& Güsten, R. 1990, ApJ, 356, 513

Stutz, A. M., \& Kainulainen, J. 2015, A\&A, 577, L6

Tafalla, M., Myers, P. C., Caselli, P., \& Walmsley, C. M. 2002, ApJ, 569, 815

Tafalla, M., Santiago-Garcia, J., Myers, P. C., et al. 2006, A\&A, 455, 577

Toci, C., \& Galli, D. 2015, MNRAS, 446, 2118

Tremblin, P., Schneider, N., Minier, V., et al. 2014, A\&A, 564, A106

Vázquez-Semadeni, E., \& Garcia, N. 2001, ApJ, 557, 727

Van Dishoeck, E. F., \& Black, 1988, ApJ, 334, 771

Ward, R. L., Wadsley, J., \& Sills, A. 2014, MNRAS, 445, 1575

Wilson, T. L., \& Rood, R. 1994, ARA\&A, 32, 191

Whitworth, A., \& Summers 1985, MNRAS, 214, 1

Wong, T., Ladd, E. F., Brisbin, D., et al. 2008, MNRAS, 386, 1069

Wright, N. J., Drake, J. J., Drew, J. E., \& Vink, J. S. 2010, ApJ, 713, 871 


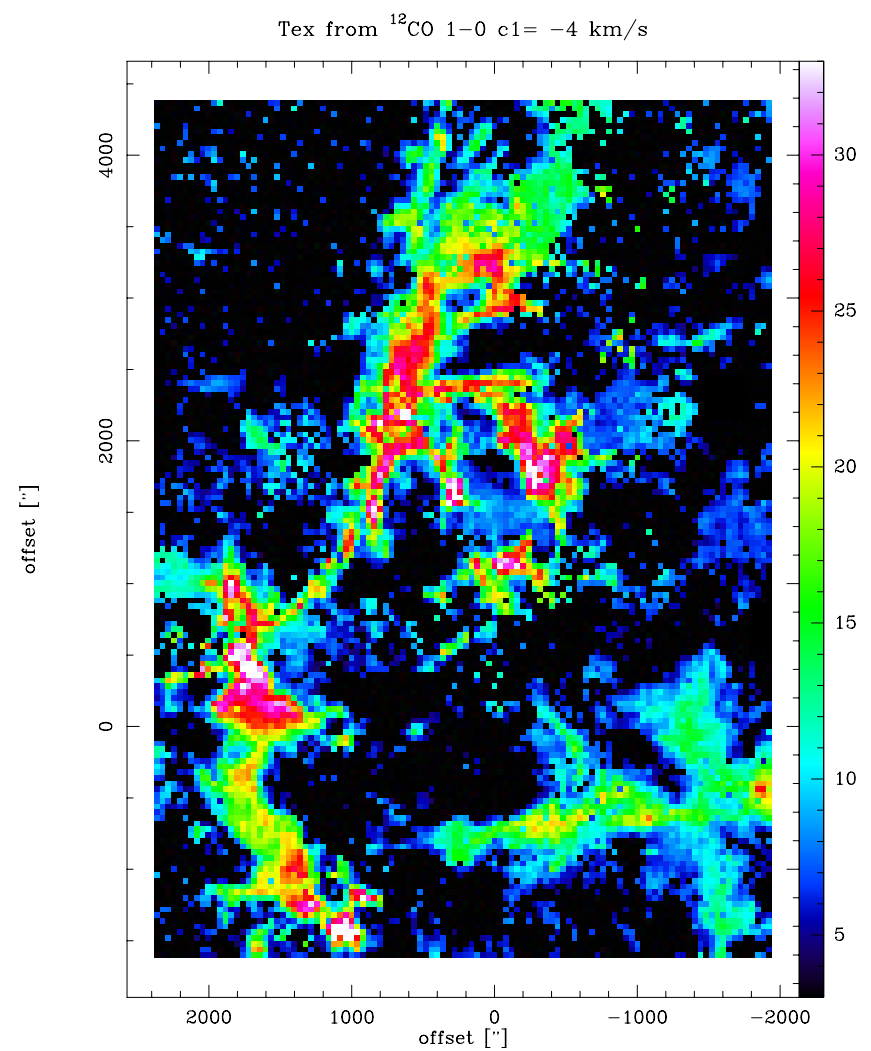

Fig. B.1. Map of excitation temperature of the main velocity component in Cygnus X North obtained from a Gaussian fit to the ${ }^{12} \mathrm{CO} 1 \rightarrow 0$ line.

\section{Appendix A: Determination of $\mathrm{H}_{2}$ column density from dust}

We assume optically thin dust emission at one dust temperature $T_{\mathrm{d}}$ for each pixel in the map and fitted a greybody function of the form $I_{v}=B_{v}\left(T_{\mathrm{d}}\right) \kappa \Sigma$ to the absolutely calibrated datapoints at $160 \mu \mathrm{m}, 250 \mu \mathrm{m}, 350 \mu \mathrm{m}$, and $500 \mu \mathrm{m}$. The specific dust opacity per unit mass (dust+gas) is approximated by the power law $\kappa_{v}=$ $0.1(v / 1000 \mathrm{GHz})^{\beta} \mathrm{cm}^{2} / \mathrm{g}$ with $\beta=2$, and the dust temperature and surface density distribution $\Sigma$ are left as free parameters. The $\mathrm{H}_{2}$ column density $N_{\text {dust }}$ is then calculated from $\Sigma=\mu_{\mathrm{H}} m_{\mathrm{H}} N_{\text {dust }}$, adopting a mean molecular weight per hydrogen molecule $\mu_{\mathrm{H}}=$ 2.8. For more details, see Hill et al. (2011). We approximate the final uncertainties in the dust column-density maps to be around $\sim 30-50 \%$, mainly as a result of the uncertainty in the assumed form of the opacity law and possible temperature gradients along the line of sight.

\section{Appendix B: Determination of $\mathrm{H}_{2}$ column density from molecular lines}

\section{B.1. Determination of and constraints on the excitation temperature}

The temperature stucture in the Cygnus X North region is complex. Large-scale UV illumination from the central Cyg OB2 cluster heats the diffuse gas and PDR surfaces. Internal (proto)stars and clusters such as DR21 show up as local hotspots. In parallel, the Cygnus X North clouds also contain a large reservoir of cold and dense gas, seen in submm continuum (Motte et al. 2007).

The observations of several molecular lines enable us to trace sensitively this temperature structure. If the LTE assumption is valid and the density is larger than the critical density of the transition, the kinetic temperature of the gas $\left(T_{\mathrm{kin}}\right)$ corresponds to the excitation temperature $T_{\text {ex }}$ for the molecular lines (which is assumed to be equal for all species). If gas and dust are well mixed, this temperature should also correspond to the dust temperature $T_{\text {dust }}$ that was derived from Herschel. However, LTE conditions are not present and the density varies from low values in the diffuse gas phase, mainly seen in ${ }^{12} \mathrm{CO}$, to high values in dense clumps in the molecular clouds, traced by dust and the $\mathrm{CS}$ and $\mathrm{N}_{2} \mathrm{H}^{+}$lines. In the following, we determine the excitation temperature in different ways, using the observational data sets.

$T_{\text {ex }}$ from ${ }^{12} \mathrm{CO} 1 \rightarrow 0$

From ${ }^{12} \mathrm{CO} 1 \rightarrow 0$, we calculate the excitation temperature assuming that this line is optically thick so that

$$
T_{\mathrm{ex}}\left({ }^{12} \mathrm{CO}\right)=\frac{5.53}{\ln \left(1+5.53 /\left(T_{\mathrm{mb}}\left({ }^{12} \mathrm{CO}\right)+0.818\right)\right)} .
$$

The molecular clouds in the Cygnus region consist of several velocity components (Schneider et al. 2006). We performed a Gaussian line fit to the four main lines (see explanations for the ${ }^{13} \mathrm{CO}$ column density). Figure B. 1 shows the resulting excitation temperature for the most prominent component at velocities around $-4 \mathrm{~km} \mathrm{~s}^{-1}$. It becomes obvious that the temperature varies significantly between $\sim 5$ and $\sim 40 \mathrm{~K}$.

\section{$T_{\text {ex }}$ from $\mathrm{N}_{2} \mathrm{H}^{+} 1 \rightarrow 0$}

We calculate the excitation temperature from $\mathrm{N}_{2} \mathrm{H}^{+}$by fitting its hyperfine structure with the known relative intensities of the seven components. The fit delivers simultaneously the opacity $\tau$ and $T_{\mathrm{ex}}$ and shows no anomaly in the relative intensities. The average value of $T_{\mathrm{ex}}$ is $7 \mathrm{~K}$ (in a range of 5 to $20 \mathrm{~K}$ ) and the opacity is generally below one. However, we emphasise that only the product $\tau \times T_{\text {ex }}$ is constrained by the fit so that the excitation temperature is not well constrained.

\section{$T_{\text {ex }}$ from dust}

The right-hand panel of Fig. 6 shows the dust temperature we derive from the SED fit to the Herschel fluxes. The average value for the dust temperature across the map is $15 \mathrm{~K}$, with a variation between 10 and $25 \mathrm{~K}$ (see also Hennemann et al. 2012). Because the dust temperature is an average along the line of sight, it is not well determined in regions with strong temperature gradients. However, for cold, dense clumps and cores that are associated with $\mathrm{CS}$ and $\mathrm{N}_{2} \mathrm{H}^{+}$emission, we expect a much smaller variation in temperature.

In summary, considering the excitation temperatures determined from the molecular lines and the dust, we use $10 \mathrm{~K}$ in the following as the best compromise for the determination of column densities. However, we also discuss other methods (using $T_{\text {ex }}$ from ${ }^{12} \mathrm{CO}$ ) to obtain the ${ }^{13} \mathrm{CO}$ column density, and study the influence of different $T_{\mathrm{ex}}$ for the $\mathrm{N}_{2} \mathrm{H}^{+}$column density.

\section{B.2. Column densities from linear molecules}

The beam-averaged total column density $N$ of any optically thin molecule can be determined from the observed line-integrated main-beam brightness temperature $T_{\mathrm{mb}}$ with

$N\left[\mathrm{~cm}^{-2}\right]=f\left(T_{\mathrm{ex}}\right) \int T_{\mathrm{mb}}[K] \mathrm{d} v\left[\mathrm{~km} \mathrm{~s}^{-1}\right]$ 

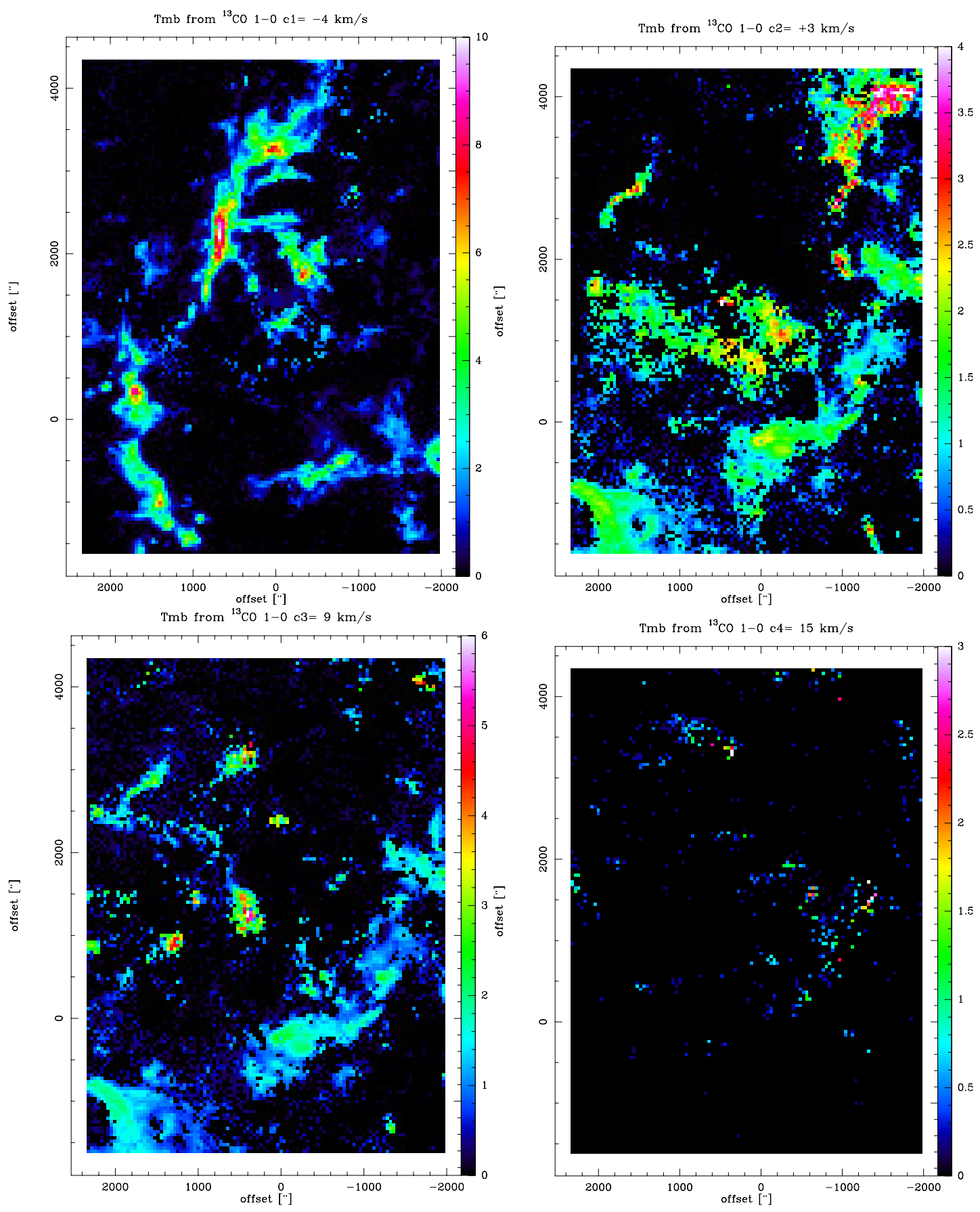

Fig. B.2. Maps of the Gaussian fits to the main-beam brightness temperature of ${ }^{13} \mathrm{CO} 1 \rightarrow 0$ for the four main velocity ranges in Cygnus $\mathrm{X}$ North. The most prominent emission comes from the velocity component at $-4 \mathrm{~km} \mathrm{~s}^{-1}$.

and for linear molecules

$$
f\left(T_{\mathrm{ex}}\right)=\frac{3 h Z}{8 \pi^{3} \mu^{2} J_{\mathrm{u}}} \frac{\exp \left(E_{\mathrm{up}} / k T_{\mathrm{ex}}\right)}{\left[1-\exp \left(-h v / k T_{\mathrm{ex}}\right)\right]\left(J\left(T_{\mathrm{ex}}\right)-J\left(T_{\mathrm{BG}}\right)\right)}
$$

and

$$
J\left(T_{\text {ex }}\right)=\frac{h v}{k\left(\exp \left(h v /\left(k T_{\text {ex }}\right)-1\right)\right.}
$$

and $J\left(T_{\mathrm{BG}}\right)=J(2.7 \mathrm{~K})$, and in which $h$ and $k$ denote the Planck and the Boltzman constants, respectively, $E_{\mathrm{up}}$ is the energy of the upper level, $v$ is the frequency [GHz], $\mu$ is the dipole moment
[Debye], $J_{\mathrm{u}}$ is the upper value of the rotational quantum number and $\int T_{\mathrm{mb}} \mathrm{d} v$ is the velocity integrated line intensity on a main beam temperature scale.

The first two terms of the rotational partition function for a diatomic linear molecule which is accurate to $1 \%$, compared to the full term for temperatures above $2 \mathrm{~K}$ (Mangum \& Shirley 2015), are given by

$$
Z=\frac{k T_{\mathrm{ex}}}{h B}+1 / 3,
$$

with the rotational constant $\mathrm{B}$ expressed to first order as $v=$ $2 \mathrm{BJ}_{\mathrm{u}}$. 


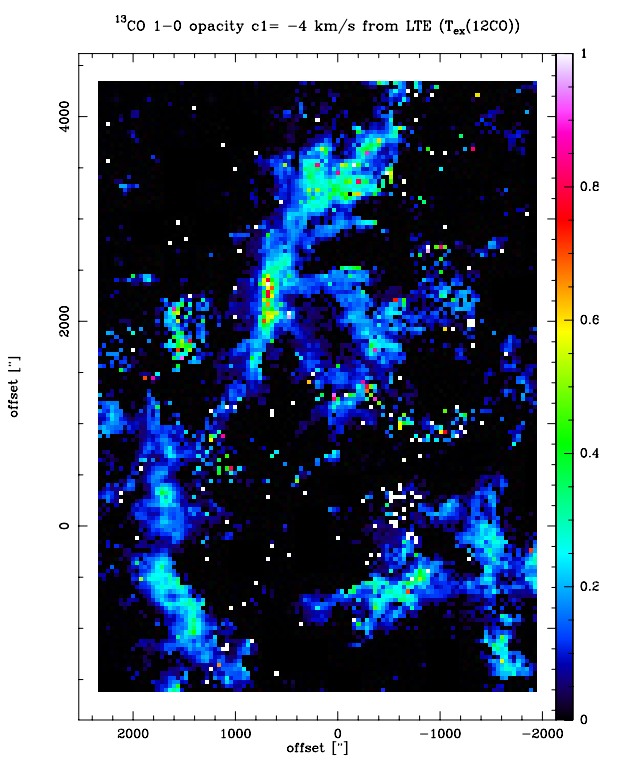

Fig. B.3. Map of the ${ }^{13} \mathrm{CO}$ opacity derived for the velocity component $-4 \mathrm{~km} \mathrm{~s}^{-1}$. White pixels indicate positions that were not well-fitted (e.g. FWHM too large, intensities too low) and thus attributed a blanking value.

To determine all $\mathrm{H}_{2}$ column densities, we apply a correction to the hydrogen mass of a factor of 1.36 to account for helium and other heavy elements.

\section{$\mathrm{H}_{2}$ column density from ${ }^{13} \mathrm{CO} \mathrm{l} \rightarrow 0$}

The values for the ${ }^{13} \mathrm{CO} 1 \rightarrow 0$ transition are $v=110.201 \mathrm{GHz}$, $\mathrm{h} v / k=5.29 \mathrm{~K}, \mu=0.112$ Debye, $J_{\mathrm{u}}=1$, and $J(2.7 K)=0.868$. The temperature-dependent factor $\mathrm{f}\left(T_{\mathrm{ex}}\right)$ is $3.22,2.00,1.62$, 1.58 , and $1.70 \times 10^{15} \mathrm{~K}^{-1}\left(\mathrm{~km} \mathrm{~s}^{-1}\right)^{-1} \mathrm{~cm}^{-2}$ for $5,7,10,15$, and $20 \mathrm{~K}$, respectively. As discussed above, we use a constant value of $10 \mathrm{~K}$ for $T_{\mathrm{ex}}$. A change in the excitation temperature between 5 and $15 \mathrm{~K}$ implies only a $10 \%$ variation in the column-density value. We then use an abundance $\left[{ }^{12} \mathrm{C}\right] /\left[{ }^{13} \mathrm{C}\right]=70$, determined from Wilson \& Rood (1994). This value corresponds well to the one derived for Orion A (Langer \& Penzias 1990). For the $\left[\mathrm{H}_{2}\right] /\left[{ }^{12} \mathrm{CO}\right]$ abundance, we use a value of $1.1 \times 10^{4}$ (Pineda et al. 2010; Fontani et al. 2012).

Because it is known that the ${ }^{13} \mathrm{CO} 1 \rightarrow 0$ line is not always optically thin, an opacity correction $\tau_{13} /\left(1-\exp \left(\tau_{13}\right)\right)$ for moderate $\tau$ can be applied for the column density (Frerking et al. 1982). To verify if this kind of correction is required, we determine the ${ }^{13} \mathrm{CO}$ opacity by using the excitation temperature $T_{\mathrm{ex}}$ that was obtained from ${ }^{12} \mathrm{CO}$ and the observed ${ }^{13} \mathrm{CO}$ main-beam brightness temperature $T_{\mathrm{mb}}\left({ }^{13} \mathrm{CO}\right)$ with

$\tau_{13}=-\ln \left(1-T_{\mathrm{mb}}\left({ }^{13} \mathrm{CO}\right) /\left(5.29 /\left(\exp \left(5.29 / T_{\mathrm{ex}}\right)-1\right)-0.868\right)\right)$.

Because the molecular clouds in the Cygnus region consist of several velocity features (Schneider et al. 2006), we perform a Gaussian line fit to four components for ${ }^{12} \mathrm{CO}$ and ${ }^{13} \mathrm{CO}$ (see B.1). As shown in Fig. B.2, the dominating emission comes from the component at $-4 \mathrm{~km} \mathrm{~s}^{-1}$, which is associated with the molecular clouds of DR21, DR22, and DR23. The component at $9 \mathrm{~km} \mathrm{~s}^{-1}$ becomes important for the W75 region and southwest of the DR21 ridge. Emission at 3 and $15 \mathrm{~km} \mathrm{~s}^{-1}$ is more diffuse and on a low intensity level. We calculate the ${ }^{13} \mathrm{CO}$ opacity for all velocity components and find that the line is optically thin everywhere, even for the $-4 \mathrm{~km} \mathrm{~s}^{-1}$ component that is shown in
Fig. B.3. We thus did not include an opacity correction for the determination of the ${ }^{13} \mathrm{CO}$ column density.

The N-PDFs that were determined from the ${ }^{13} \mathrm{CO}$ column density, using a constant excitation temperature of $10 \mathrm{~K}$ or a variable pixel-to-pixel temperature $T_{\mathrm{ex}}$ from ${ }^{12} \mathrm{CO}$, do not show a significant difference.

\section{$\mathrm{H}_{2}$ column density from $\mathrm{C}^{18} \mathrm{O} 1 \rightarrow 0$}

The values for the $\mathrm{C}^{18} \mathrm{O} 1 \rightarrow 0$ transition are $v=109.782 \mathrm{GHz}$, $\mathrm{h} v / k=5.27 \mathrm{~K}, \mu=0.1098$ Debye, $J_{\mathrm{u}}=1$, and $J(2.7 \mathrm{~K})=$ $0.872 \mathrm{~K}$. The temperature-dependent factor $\mathrm{f}\left(T_{\mathrm{ex}}\right)$ is $3.34,2.08$, 1.69, 1.65, and $1.78 \times 10^{15} \mathrm{~K}^{-1}\left(\mathrm{~km} \mathrm{~s}^{-1}\right)^{-1} \mathrm{~cm}^{-2}$ for 5, 7, 10, 15 , and $20 \mathrm{~K}$, respectively. As discussed in Sect. B.1, we adopt a value of $10 \mathrm{~K}$ for $T_{\mathrm{ex}}$. To determine the $\mathrm{H}_{2}$ column density, we then use a ratio $\left[\mathrm{H}_{2}\right] /\left[{ }^{12} \mathrm{CO}\right]$ of $1.1 \times 10^{4}$ (Pineda et al. 2010; Fontani et al. 2012) and an abundance $\left[{ }^{16} \mathrm{O}\right] /\left[{ }^{18} \mathrm{O}\right]=531$, which was determined from Wilson \& Rood (1994), using a distance of $1.4 \mathrm{kpc}$.

We determine the $\mathrm{C}^{18} \mathrm{O}$ opacity in the same way for the four line components as we did for ${ }^{13} \mathrm{CO}$ (see above), using the excitation temperature $T_{\text {ex }}$ obtained from ${ }^{12} \mathrm{CO}$ and the observed $\mathrm{C}^{18} \mathrm{O}$ main-beam brightness temperature $T_{\mathrm{mb}}\left(\mathrm{C}^{18} \mathrm{O}\right)$ with

$\tau_{18}=-\ln \left(1-T_{\mathrm{mb}}\left(\mathrm{C}^{18} \mathrm{O}\right) /\left(5.27 /\left(\exp \left(5.27 / T_{\mathrm{ex}}\right)-1\right)-0.87\right)\right) .($

Maps of the resulting $\mathrm{C}^{18} \mathrm{O}$ opacity show that the line is optically thin for all velocity components and reaches its maximum value of $\tau_{18} \sim 0.4$ only in the DR2 1 ridge for the $-4 \mathrm{~km} \mathrm{~s}^{-1}$ component.

\section{$\mathrm{H}_{2}$ column density from ${ }^{12} \mathrm{CO} \mathrm{l} \rightarrow 0$}

The ${ }^{12} \mathrm{CO} 1 \rightarrow 0$ line is optically thick so that it should a priori not be a good tracer for the total column density. However, empirical studies (Strong et al. 1988; Bolatto et al. 2013) show that the line still can trace the total column density/mass of a molecular cloud reasonably well with a conversion factor of $2-2.3 \times 10^{20}$ from line integrated $\mathrm{CO}$ main-beam brightness temperature into $\mathrm{H}_{2}$ column density. Here, we adopt a value of $2 \times 10^{20} \mathrm{~K}^{-1}$ $\left(\mathrm{km} \mathrm{s}^{-1}\right)^{-1} \mathrm{~cm}^{-2}$.

$\mathrm{H}_{2}$ column density from $\mathrm{CS} 2 \rightarrow 1$

The values for the CS $2 \rightarrow 1$ transition are $v=98.0 \mathrm{GHz}$, $\mathrm{h} v / k=4.70 \mathrm{~K}, \mu=1.95$ Debye, $J_{\mathrm{u}}=2$, and $J(2.7 \mathrm{~K})=1.00$. The factor $f\left(T_{\mathrm{ex}}\right)$ is $16.1,9.48,7.35,7.00$, and $7.41 \times 10^{12} \mathrm{~K}^{-1}$ $\left(\mathrm{km} \mathrm{s}^{-1}\right)^{-1} \mathrm{~cm}^{-2}$ for $5,7,10,15$, and $20 \mathrm{~K}$. We assume optically thin $\mathrm{CS}$ emission and an abundance $[\mathrm{CS}] /\left[\mathrm{H}_{2}\right]$ of $4 \times 10^{-10}$ (Fechtenbaum et al., in prep.).

\section{$\mathrm{H}_{2}$ column density from $\mathrm{N}_{2} \mathrm{H}^{+} \mathrm{l} \rightarrow 0$}

The values for the $\mathrm{N}_{2} \mathrm{H}^{+} 1 \rightarrow 0$ transition are $v=93.17 \mathrm{GHz}$, $\mathrm{h} v / k=4.47 \mathrm{~K}, \mu=3.40$ Debye, $J_{\mathrm{u}}=1$, and $J(2.7 \mathrm{~K})=1.06$. The factor $f\left(T_{\mathrm{ex}}\right)$ is $3.62,2.44,2.10,2.16$, and $2.39 \times 10^{12} \mathrm{~K}^{-1}$ $\left(\mathrm{km} \mathrm{s}^{-1}\right)^{-1} \mathrm{~cm}^{-2}$ for $5,7,10,15$, and $20 \mathrm{~K}$. The total beam averaged $\mathrm{N}_{2} \mathrm{H}^{+}$column density is calculated using the line-integrated emission from the $F=(2,1) J=(1,0)$ component at a frequency of $93.1737767 \mathrm{GHz}$ and a HFS intensity ratio of 7/27 for that component. We assume an abundance $\left[\mathrm{N}_{2} \mathrm{H}^{+}\right] /\left[\mathrm{H}_{2}\right]$ of $5 \times 10^{-10}$.

We emphasise that all $\mathrm{H}_{2}$ column-density determinations have a large uncertainty, mainly arising from the abundance. The $[\mathrm{CO}] /\left[\mathrm{H}_{2}\right]$ abundance for the $\mathrm{CO}$ isotopolgues is probably better constrained because it has been well studied in the literature. However, the variation in excitation temperature along the line of sight is more significant here because the $\mathrm{CO}$ lines trace a larger range in densities and temperatures than the high-density tracers 

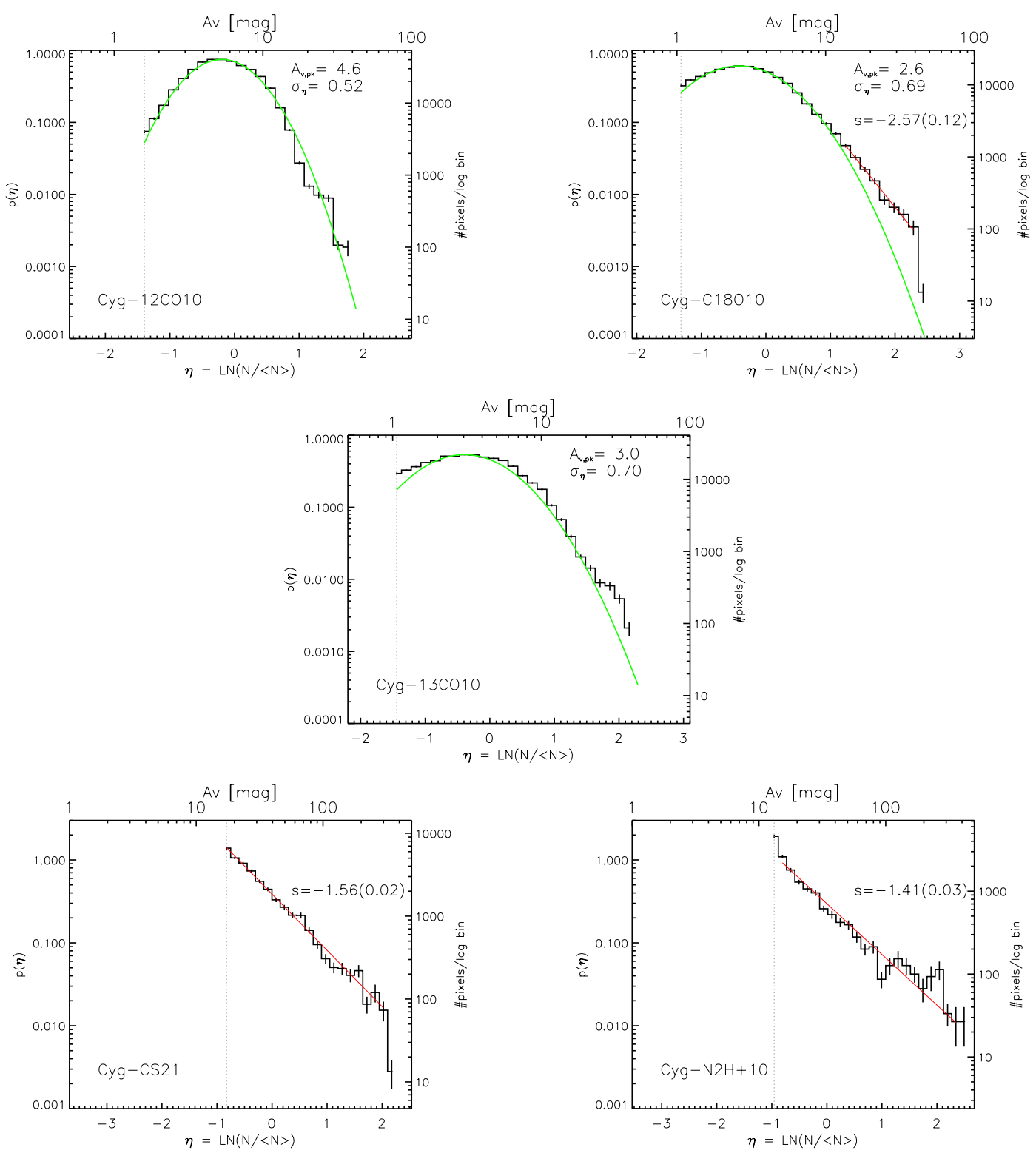

Fig. C.1. PDFs derived from the molecular line maps. The left $y$-axis gives the normalized probability $p(\eta)$, the right $y$-axis the number of pixels per log bin. The upper $x$-axis is the visual extinction and the lower $x$-axis the logarithm of the normalized column density. For the CO lines, we fitted a lognormal to the low column-density range, indicated by a green curve. The red line shows the power-law fit to the high column-density end for $\mathrm{C}^{18} \mathrm{O}, \mathrm{CS}$, and $\mathrm{N}_{2} \mathrm{H}^{+}$. The respective slopes $s$ (and errors) are given in the panels. For the CO lines, the peak and width of the PDF is indicated with $A_{v \text { peak }}$ and $\sigma$.

$\mathrm{CS}$ and $\mathrm{N}_{2} \mathrm{H}^{+}$. For the latter, the constant excitation temperature assumption is most likely more valid, but the abundances of $\mathrm{CS}$ and $\mathrm{N}_{2} \mathrm{H}^{+}$are less well constrained, in particular for massive clumps and cores. In summary, we consider the $\mathrm{H}_{2}$ column densities from $\mathrm{CO}$ to be correct within a factor of two and the ones from $\mathrm{CS}$ and $\mathrm{N}_{2} \mathrm{H}^{+}$within a factor of a few.

\section{Appendix C: Individual molecular line PDFs}

In the main body of the paper, we used a normalization for the PDF with respect to the size, i.e. pixel number of the dust map. We here show the PDFs obtained with a normalization $\int_{-\infty}^{+\infty} p_{\eta} \mathrm{d} \eta=\int_{0}^{+\infty} p_{N} \mathrm{~d} N=1$. As such, the PDFs are consistent with what is shown in the literature (see Schneider et al. 2015a, and references therein) and the number of pixels defining the PDF can be extracted. Figure C. 1 shows the resulting PDFs.

\section{Appendix D: Relation between PDF slope and radial density distribution for different geometries}

We define the PDF $p_{N}$ as the surface fraction with gas of column density $N$ and the PDF of the natural log of the column density density $p_{\eta}$ with $\eta=\ln (N /\langle N\rangle)$, the relation between the two is $p_{\eta}=N p_{N}$. The definition of $p_{N}$ gives $p_{N} \propto \mathrm{d} A / \mathrm{d} N$.

Spherical geometry

In the case of spherical geometry, the density radial profile is $\rho(r) \propto r^{-\alpha}$, hence $N \propto \rho r \propto r^{-\alpha+1}\left(\mathrm{~d} N \propto r^{-\alpha}\right)$. Hence, with the area $A \propto r^{2}(\mathrm{~d} A \propto r)$

$p_{N} \propto \mathrm{d} A / \mathrm{d} N \propto r / r^{-\alpha} \propto N^{(1+\alpha) /(1-\alpha)}$
$p_{\eta}=N p_{N} \propto N^{2 /(1-\alpha)}$

Cylindrical geometry

In the case of cylindrical geometry, the density radial profile from the axis of the cylinder is $\rho\left(r_{\mathrm{c}}\right) \propto r_{\mathrm{c}}^{-\alpha}$, hence $N \propto \rho r_{\mathrm{c}} \propto$ 
N. Schneider et al.: Understanding star formation in molecular clouds. III.

$r_{\mathrm{c}}^{-\alpha+1}$. However in this case $A \propto z \times r_{\mathrm{c}} \propto r_{\mathrm{c}}$, hence

$p_{N} \propto \mathrm{d} A / \mathrm{d} N \propto 1 / r_{\mathrm{c}}^{-\alpha} \propto N^{\alpha /(1-\alpha)}$

$p_{\eta}=N p_{N} \propto N^{1 /(1-\alpha)}$.

Note that similar calculations can be found in Federrath

\& Klessen (2013), Fischera et al. (2014), and Myers (2015). 